\title{
HARD DRIVE COMMAND CAPTURE AND SEQUENTIAL STREAM DETECTION
}

\author{
A Thesis \\ Presented to \\ the Faculty of California Polytechnic State University \\ San Luis Obispo
}

\author{
In Partial Fulfillment \\ of the Requirements for the Degree \\ Master of Science in Computer Science
}

by

Adam Miller

June 2012 
(C) 2012

Adam Miller

ALL RIGHTS RESERVED 


\section{COMMITTEE MEMBERSHIP}

TITLE: Hard Drive Command Capture and Sequential Stream Detection

AUTHOR: $\quad$ Adam Miller

DATE SUBMITTED: June 2012

COMMITTEE CHAIR: Chris Lupo, Ph.D.

COMMITTEE MEMBER: John Oliver, Ph.D.

COMMITTEE MEMBER: Phillip Nico, Ph.D. 


\begin{abstract}
Hard Drive Command Capture and Sequential Stream Detection
\end{abstract}

Adam Miller

This thesis explores hardware command capture as a viable means of analyzing real world hard drive usage. Hardware command capture provides insight into the IO stack where current tools fail to reach. A software platform is presented which provides trace conversion and analysis capabilities. This platform is written in Python and designed to handle traces of arbitrary size while being easily extensible for future projects to build upon. A novel Sequential Stream Detection algorithm built upon the software platform is then presented. This algorithm detects application level sequential streams and provides interesting insight into the sequential nature of the applications analyzed. The software platform and Sequential Stream Detector were validated and run against a range of workloads including video playback, large project compilations, and synthetic benchmarks. Where applicable, each workload was run on multiple file systems (ext2, ext3, ext4, Btrfs) to compare the effects of stream allocation across file systems. It is shown that stream allocation is consistent across file systems suggesting stream detection may be a valuable workload identification tool. 


\section{Acknowledgements}

The work in this thesis was made possible through the support of Western Digital. Western Digital provided the initial idea of exploring sequential streams as well as a wealth of knowledge, tools, and expertise. 


\section{Contents}

List of Tables viii

List of Figures $\quad$ ix

1 Introduction 1

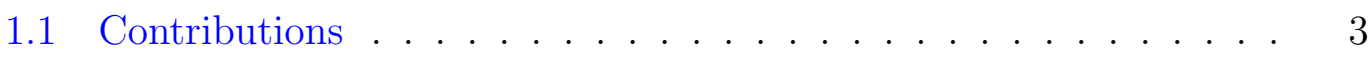

1.2 Organization ...................... 6

$\begin{array}{lll}2 & \text { Background } & 7\end{array}$

2.1 Existing Tools . . . . . . . . . . . . . . . . 7

2.1.1 Benchmarking ................ 8

2.1.2 Analytic Modeling .............. 8

2.1.3 Simulation . . . . . . . . . . . . . . 9

2.1.4 Software Tracing . . . . . . . . . . . . . . 10

2.2 Why Hardware Tracing? . . . . . . . . . . . . . . . . . . . . . . 10

3 Trace Analysis Platform $\quad 13$

3.1 Overview . . . . . . . . . . . . . . . . . . 13

3.2 TraceToJson . . . . . . . . . . . . . . . . 15

3.3 PyTrace . . . . . . . . . . . . . . . . . . 16

3.3.1 JSONReader . . . . . . . . . . . . . . 16

3.3.2 SATA Command Parser . . . . . . . . . . 16

3.3.3 Command Statistics Generator . . . . . . . . . . . . 18

3.3.4 Memory Usage . . . . . . . . . . . . . . . . . 19

3.4 Database .......................... 20

4 Sequential Stream Detection $\quad 23$ 
4.1 The Case for Observing Sequential Streams . . . . . . . . . . . 23

4.2 Formal Definition of a Sequential Stream . . . . . . . . . . . . . . 25

4.3 Window Size . . . . . . . . . . . . . . . 26

4.4 A Sequential Stream Detection Algorithm . . . . . . . . . . . . 32

4.5 Implementation . . . . . . . . . . . . . . . . . . 35

$\begin{array}{lll}5 & \text { Validation } & 37\end{array}$

5.1 Trace Analysis Platform Validation . . . . . . . . . . . . . . . 37

5.2 Sequential Stream Detection Validation . . . . . . . . . . . . . 38

5.2.1 Workloads Run . . . . . . . . . . . . . . . 38

5.2.2 Results...................... 39

6 Trace Analysis Using Sequential Stream Detection 42

6.1 Stream Graphs . . . . . . . . . . . . . . . . . . . . . . . . . . 42

$6.2 \mathrm{dd} \ldots \ldots \ldots \ldots . \ldots \ldots 4 . \ldots \ldots$

6.3 mplayer . . . . . . . . . . . . . . . . . . 49

6.4 Trace Fingerprinting . . . . . . . . . . . . . . . . 53

7 Future Work $\quad 60$

8 Conclusion $\quad 62$

$\begin{array}{ll}\text { Bibliography } & 64\end{array}$ 


\section{List of Tables}

3.1 Memory Usage . . . . . . . . . . . . . . . . . . . . . . 20

4.1 Sequential Stream Detection Runtimes. Window Size 100ms . . . 36

5.1 Sequential Stream Detection Validation Results . . . . . . . . . 39

5.2 Sequential Stream Detection Incorrect Window Size . . . . . . . 41 


\section{List of Figures}

1.1 Contribution Flow . . . . . . . . . . . . . . 5

2.1 Hardware vs Software Tracing . . . . . . . . . . . . . . . 12

3.1 Trace Analysis Platform Overview . . . . . . . . . . . . . . . 14

4.1 Disk Access Scenarios . . . . . . . . . . . . . . . . . . 25

4.2 mplayer video playback on Btrfs. Window size 1 s $\ldots \ldots \ldots . .27$

4.3 mplayer video playback on Btrfs. Window size 10s . . . . . . . 28

4.4 fio mixed sequential and random on the raw block device . . . . 30

4.5 fio mixed sequential and random on the raw block device. Window size 10 s. . . . . . . . . . . . . . . . . . 31

4.6 Sequential Stream Detector _. . . . . . . . . . . . . . 36

6.1 Fedora 16 GCC RPM rebuild on Btrfs - Streams . . . . . . . . . 44

6.2 Fedora 16 GCC RPM rebuild on Btrfs - LBA . . . . . . . . 45

6.3 Fedora 16 GCC RPM rebuild on Btrfs - LBA and Streams . . . 46

$6.4 \mathrm{dd}$ if $=/ \mathrm{dev} /$ urandom of $=/ \mathrm{dev} / \mathrm{sdb} 1 \mathrm{bs}=4 \mathrm{~K} \ldots \ldots \ldots \ldots$

6.5 mplayer video playback on Btrfs. LBA as a function of time . . 50

6.6 mplayer video playback on Btrfs. Window size 10s . . . . . . . 51

6.7 mplayer video playback on Btrfs. LBA and Streams . . . . . . . 52

6.8 Fedora 16 GCC RPM rebuild on Btrfs - LBA . . . . . . . . 54

6.9 Rebuild of Fedora 16 GCC RPM on ext2. LBA as a function of time 55

6.10 Rebuild of Fedora 16 GCC RPM on ext2. LBA range restricted to $[0,25000000] \ldots \ldots \ldots \ldots \ldots \ldots \ldots$ 
6.11 Rebuild of Fedora 16 GCC RPM on ext2. Window size 10s . . . . 58

6.12 Rebuild of Fedora 16 kernel RPM on ext2. Window size 10s . . . 59 


\section{Chapter 1}

\section{Introduction}

Arnold Allen describes computer performance as follows: The word performance in computer performance means the same thing that performance means in other contexts, that is, it means "How well is the computer doing the work it is supposed to do?"[1]. This statement summarizes the end goal of all computer performance analysis; determining how well the computer is performing a certain task. As a complex system however, it can be difficult to predict or measure the performance of a computer on any particular workload. There are many metrics by which some representation of performance can be measured (latency, throughput, instructions per second, power consumption, etc)[1] but not all are applicable to every workload. To add to the complexity of selecting the correct metrics by which to judge performance is the equally daunting, if not more daunting, task of gathering the correct data from the correct sources to build the metrics. Computer performance analysis therefore comes down to selecting the metrics which are actually useful in demonstrating how effectively work is being done, gathering the data necessary, and drawing conclusions from the data. Fortunately, while this is a wide problem, as a system a computer is only as fast as 
its slowest component.

The slowest part of a modern computer is the hard drive. Compared to RAM which has access times in nanoseconds most modern magnetic based hard drives have access times in milliseconds. As an example, the Western Digital WD60000HLHX hard drive (10,000 RPM, 600 GB) has an average latency of 3 ms[4] while DDR3-1600 9-9-9 RAM has a theoretical CAS latency of 11.25ns[7]. Despite such a huge access time discrepancy a hard drive is necessary for many applications as a large capacity, non-volatile storage medium. This combination of inherent slowness and necessity makes the hard drive a prime candidate for investigation.

Unfortunately, to many software developers and system administrators a hard drive is a black box that stores data. Commands are issued telling the drive to store or retrieve data from a particular location (LBA - Logical Block Address) and there is little feedback aside from throughput (number of IOPS - IO Operations Per Second) and response time (time between command issue and command completion). These are useful metrics but leave much to be desired for a deep understanding of how the IO subsystems are performing. The flaw with only looking at throughput or response time is that such high level metrics fail to account for all the complexity within an IO subsystem that is composed of many layers. It is entirely possible for an IO request to travel from the user space application through kernel buffering, the file system, Logical Volume Manager (LVM)[10], and a RAID controller before finally reaching the physical disk. In this case a simple request to read a single byte from a file would be translated to an LBA by the file system, remapped to a physical LBA by LVM, translated to another LBA by the RAID controller, and finally read from disk.

Drive simulation and analytic modeling represent the current forefront of 
academic drive performance analysis $[5,18,21,23]$. In drive simulation workloads are run against a software hard drive modeling a real drive. In analytic modeling a model is built using metrics such as throughput, latency, and drive queue depth[21] to predict drive performance. Both of these approaches are subject to assumptions and simplifications which hamper their ability to accurately mimic or predict a real hard drive. Another shortcoming of these approaches is the difficulty in applying them to real world workloads. An enterprise application may be too complex or performance sensitive to apply either approach in a cost effective and realistic manner. Finally, software IO tracing tools exist[2] which provide very detailed information about the state and usage of the software IO subsystems. The problem with such tools is that they are fundamentally limited to observing the software layers; once a command is issued to the hardware all ability to track through the hardware layers is lost.

\subsection{Contributions}

To overcome the limitations of simulation, modeling, and software tracing this thesis explores capturing SATA commands using a serial bus analyzer. A physical bus analyzer is placed between a SATA drive and a running system which is capable of capturing all data going over the wire. Such captured traces do not suffer from the inherent assumptions of simulation/modeling and provide insight into the IO stack where software tracing cannot reach. This thesis provides three contributions:

- A demonstration of using bus level command capture (hardware tracing) for analysis. All traces have been captured using a JDSU Xgig Serial Bus Analyzer and processed with PyTrace. 
- A flexible trace analysis platform designed to be built for low resource utilization and student expansion.

- A sequential stream detection algorithm designed to detect application and OS level streams regardless of interleaving. 


\section{Serial Bus Analyzer}

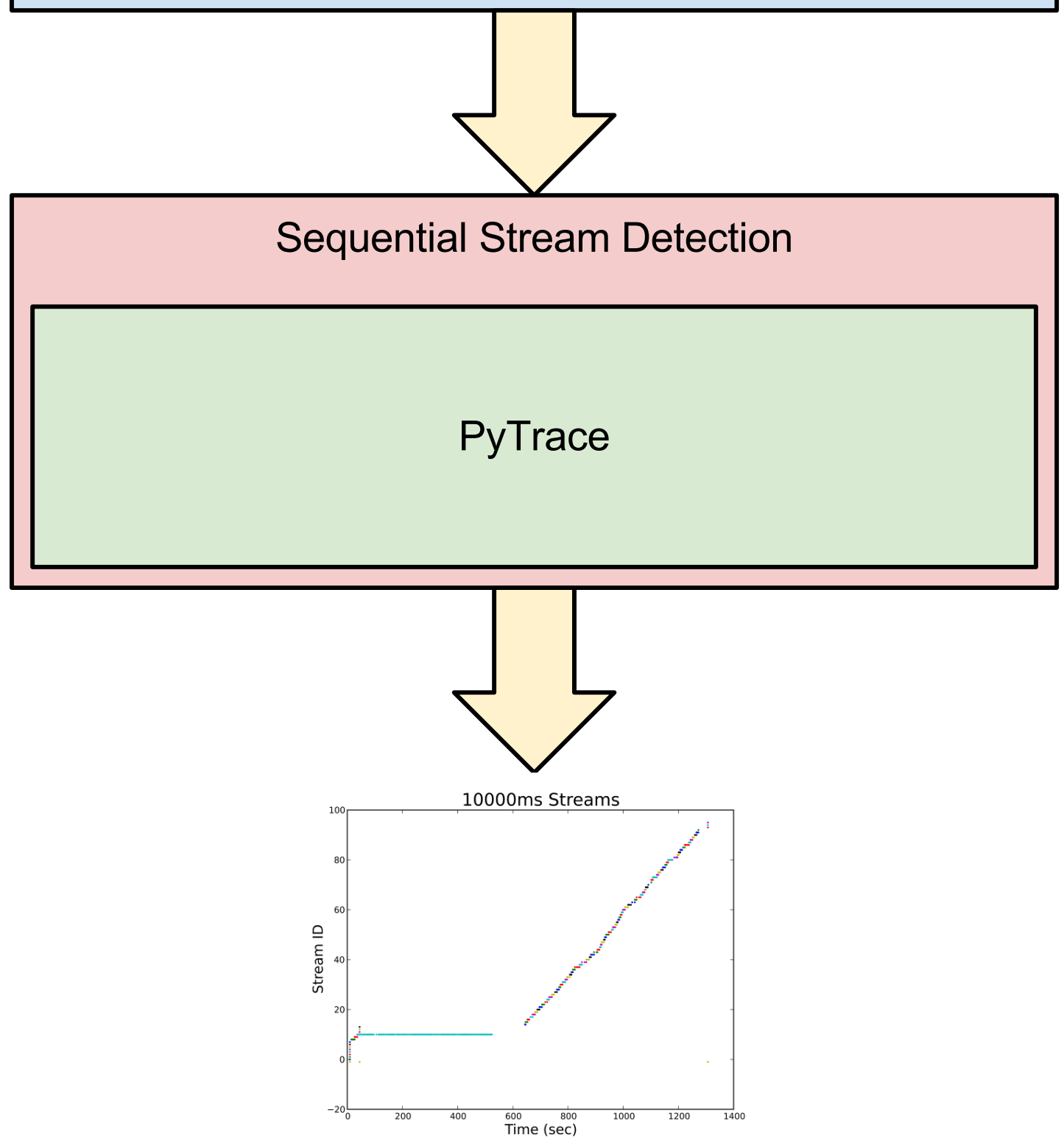

Figure 1.1: Contribution Flow 


\subsection{Organization}

The rest of the document is organized as follows. Chapter 2 discusses existing tools for hard drive analysis and observation ending with the justification for hardware tracing. Chapter 3 discusses the design and implementation of PyTrace as a platform for processing hardware traces. Chapter 4 discusses the sequential stream detection algorithm and implementation built upon PyTrace. Chapter 5 explains the validation used for PyTrace and the Sequential Stream Detector. Chapter 6 explores trace analysis using graphs produced by the Sequential Stream Detector. Chapter 7 covers possible future work and the document concludes with Chapter 8. 


\section{Chapter 2}

\section{Background}

\section{$2.1 \quad$ Existing Tools}

There is surprisingly little research utilizing SATA command capture to be found inside or outside of academia. The closest work to that presented in this thesis is done by Alma Riska and Erik Riedel. Riska and Riedel describe using capturing at the disk level to characterize the IO patterns of enterprise, desktop, and consumer electronic applications[15]. Their work corroborates previous work $[12,16,17]$ in the area of workload characterization to show that the nature of IO is highly dependent on the workload generating the IO. READ/WRITE ratio and access patterns tend to be the characteristics which vary depending on workload while certain characteristics, such as degree of IO burst, remain consistent across application and environment[15]. Instead of using command capture, most commercial and academic research into hard drive usage and performance comes in the form of benchmarking, analytic modeling, simulation, or software tracing. The rest of this chapter covers the existing tools commonly used for hard drive analysis and wraps up with the justification for hardware tracing. 


\subsubsection{Benchmarking}

Benchmarking represents the most common means of analyzing hard drive performance. Benchmarks are a low cost, easily understood measure of performance and readily available for commercial and personal use. IOMeter[6], PCMark05[14], and PassMark[13] are only three of the many benchmarking suites available. IOMeter is specifically targeted at hard drive performance measurement while PCMark05 and PassMark are suites designed to measure full system performance. Each application uses synthetic workloads as representative samples of real world work to measure and rate IO throughput and response time. The problem with benchmarks is that their accuracy depends almost entirely on how representative the synthetic workload is for the real workload[19]. A bench-

mark which simulates multimedia playback in no way demonstrates how well a hard drive will perform running a transactional database.

\subsubsection{Analytic Modeling}

Analytic modeling can be a better, more accurate predictor of performance than benchmarking while being simpler and cheaper than full blown simulation. Compared to detailed simulation, modeling also requires less knowledge of the inner works of the hard drive itself which a manufacturer is likely to keep confidential. One of the key difficulties in modeling is the state dependent and non-linear nature of a hard drive. Aspects such as seek time, rotational latency, transfer time, and bus contention are often simplified to linear functions or uniform distribution lookups despite these representations being unsuitable in many situations[18].

Wang et al. tackle this by utilizing CART (Classification And Regression 
Trees) models. CART modeling uses machine learning to approximate functions in multi-dimensional Cartesian space[22]. CART modeling is basically a form of non-linear regression, that is a means of modeling a non-linear function by mapping the relationship between dependent and independent variables. The CART model is given no details of the drive being modeled and trained for a period of time using test traces. Two different CART models are described using either IO request information or entire workload information as the input for the model. The request based model predicts latencies for each request which cumulatively represent the workload latency while the workload model predicts the latency of the workload as a whole[22]. The problem with a machine learning approach such as CART models is the dependence on accurate and extensive training traces. Training input generation is non-trivial and a diverse set of inputs must be used to provide accurate predictions for a wide range of inputs[22].

\subsubsection{Simulation}

Simulation is capable of providing more information at the cost of higher complexity compared to modeling. Modeling intentionally tries to simplify down to a single function or set of functions which represent the system while simulation tries to reproduce as many components of the hard drive as possible. The difficulty in simulation is managing the complexity and faithfully reproducing the various components and systems of IO stack.

Chris Ruemmler and John Wilkes provide a detailed description of the characteristics of modern hard drives (circa 1994) and the complexities inherent to creating models and simulations. Such complexities include accounting for drive

head positioning and for seek, rotation, and transfer times[18]. Ruemmler and 
Wilkes build upon existing simulation models which work to account for these complexities in drive simulation. Using Ruemmler and Wilkes work, Kotz et al. created a detailed simulation model of the HP 97560 Disk Drive which is capable of simulating any number of 97560 disks[9]. Finally, DiskSim represents one of the most comprehensive hard drive simulation environments available capable of simulating disks, controllers, buses, device drivers, disk block caches, and disk arrays[3].

\subsubsection{Software Tracing}

Software tracing tools such as blktrace[2] on Linux and Xperf[24] on Windows allow for fine grained capture and analysis of IO from software. The benefit of software tracing is that it doesn't suffer from the shortcomings of benchmarking, modeling, or simulation. Where the other approaches would place restrictions software tracing allows real workloads to be used on production hardware with minimal added overhead. Blktrace uses the Linux kernel's debug file system to relay and capture IO events occurring between the Linux kernel and user space. Xperf leverages Event Tracing for Windows (ETW) to generate and capture IO kernel events. Both blktrace and xperf provide supporting tools for processing and analyzing the generated traces.

\subsection{Why Hardware Tracing?}

With a variety of tools available to analyze a hard drive and the IO stack (2.1.1, 2.1.2, 2.1.3, 2.1.4) one might think there is little need for yet another tool. Even considering the deficiencies present in benchmarking (unrealistic workload), 
modeling (simplifying assumptions), and simulation (complexity, performance, and realism) it would seem software tracing provides an ideal means of observation and analysis. This is true save for two deficiencies in software tracing: software only focus and platform support.

Software tracing provides insight into the IO stack up to the point that the IO leaves the system. Such a trace is comprehensive for systems in which the kernel talks directly to the hard drive(s) but not for systems which have hardware RAID controllers or other hardware layers between the kernel and the hard drive(s). In systems such as these the command which was issued by the kernel is not necessarily the same command that is received by the drive in question. The other deficiency, platform support, rules out trying to trace any system which is incapable of running the software tracer (DVRs, Video Game Consoles, Media Centers).

Hardware tracing fills in the gaps left by software tracing. A bus analyzer, at least in theory, can be put between any two pieces of hardware in the system to capture the commands as they go over the wire. A bus analyzer is platform independent as well; requiring no software support. The only requirement to use a bus analyzer is physical access to the system in question with the added benefit of no run-time overhead for the workload being captured. In this way hardware tracing either fills in the hole left by software tracing or allows for investigation in the first place. 


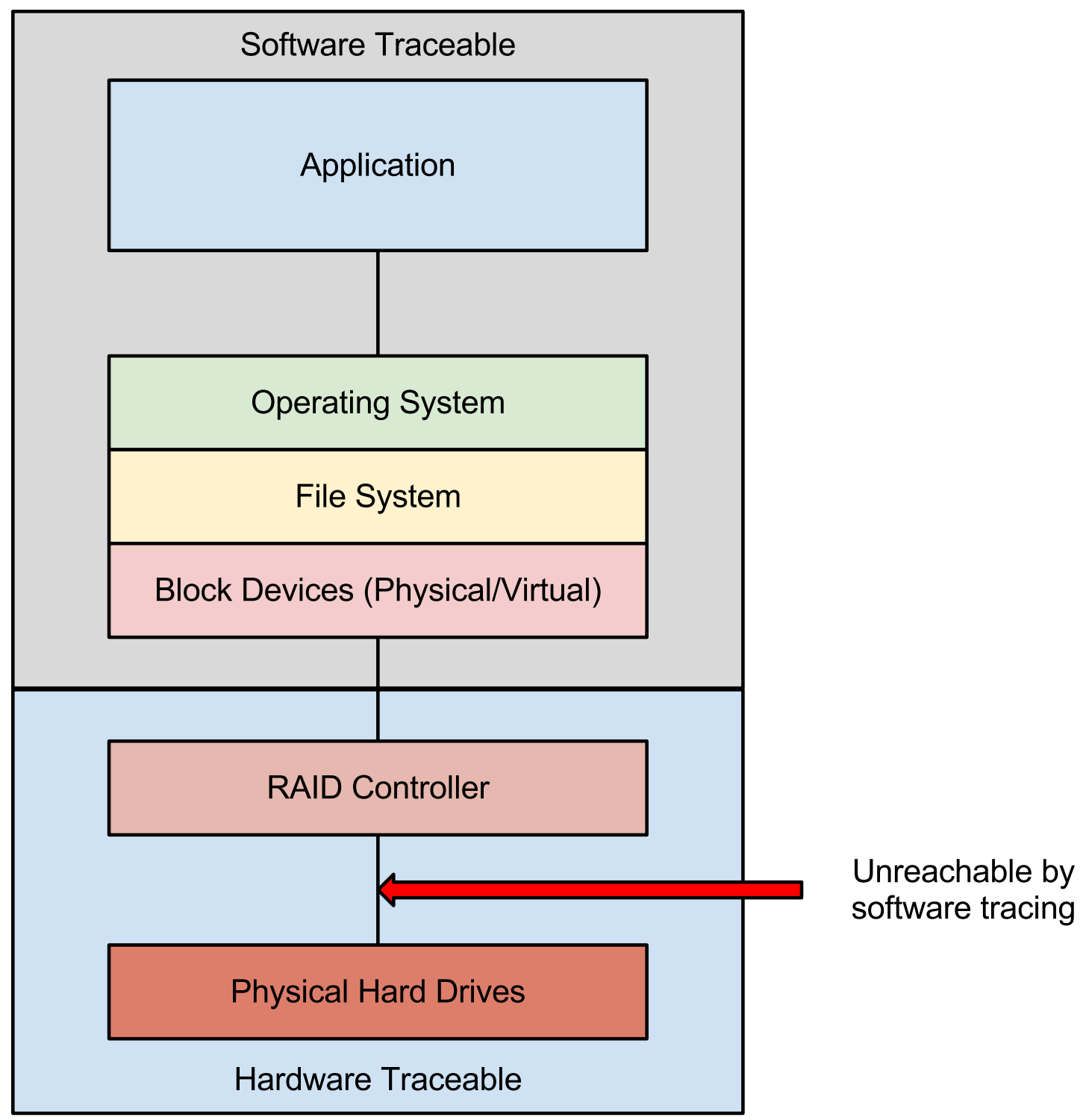

Figure 2.1: Hardware vs Software Tracing 


\section{Chapter 3}

\section{Trace Analysis Platform}

\subsection{Overview}

All the potential insight into hard drives and their usage that hardware tracing is capable of giving goes to waste without a means of processing and analyzing the traces. Unfortunately, since SATA/SAS bus analyzers are expensive, specialty items the only tools which exist for handling the captured traces are the support software produced by the analyzer manufacturer and custom solutions produced by hard drive manufacturers. Support software tends to be rigid and closely coupled with the analyzer itself while custom solutions are usually unavailable to the public. An ideal platform would be freely available and open for modification to support all manner of esoteric analyzer or analysis strategy. The components of the Trace Analysis Platform presented here, TraceToJson and PyTrace, have been written to address the shortcomings of existing tools with respect to the analysis of hardware traces in academia. 

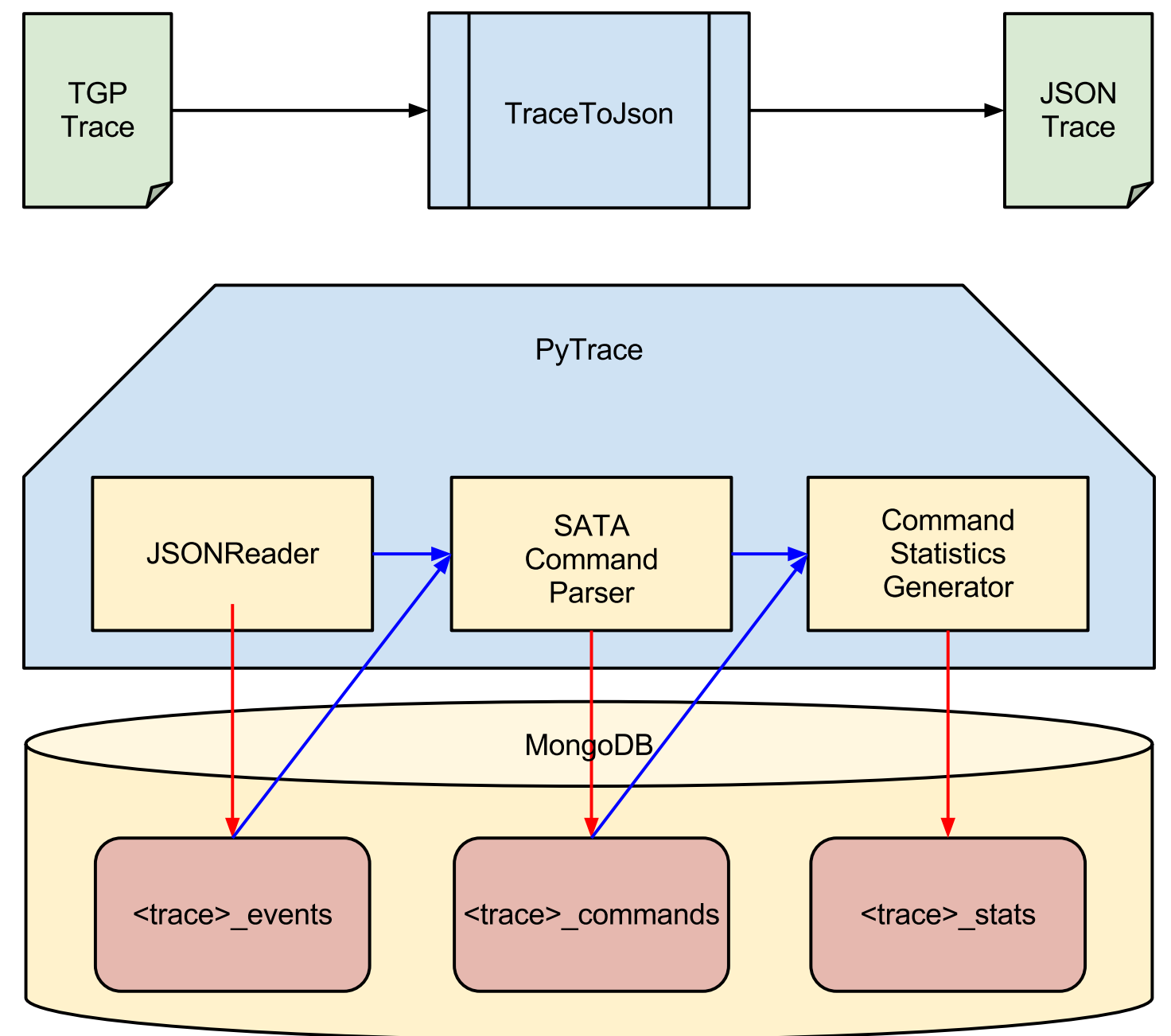

Figure 3.1: Trace Analysis Platform Overview 


\subsection{TraceToJson}

The TraceToJson tool is the simplest and most critical piece of the platform. The goal of TraceToJson is to translate a captured trace into a platform agnostic JSON representation. JSON is chosen for its wide language support and simplicity[8]. Currently TraceToJson only supports the JDSU Xgig analyzer and translates from the proprietary TGP format using the TraceDataIO.dll library.

The text file produced by TraceToJson contains newline separated trace events encoded as JSON objects. The following is an example JSON event object formatted for legibility:

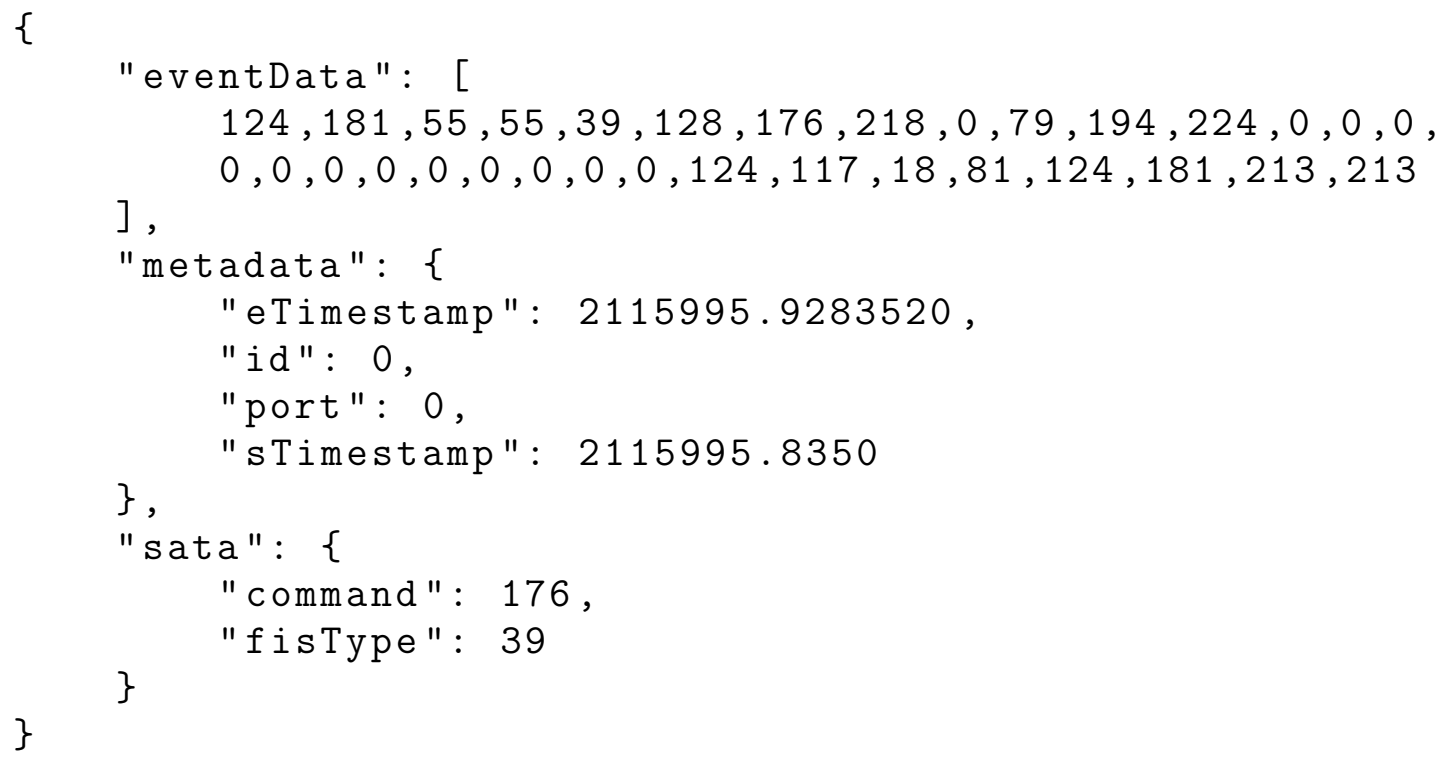

All data present in the JSON object, except for the sata field, is taken directly from the original event object. The eventData field contains the captured event data which in this example is 32 bytes of SATA command information. The metadata field contains additional metadata added to the event by the analyzer. The fields within the metadata object are start time in microseconds (sTimestamp), end time in microseconds (eTimestamp), event id $(i d)$, and analyzer port number (port). Finally the sata field is parsed from the eventData to identify the protocol 
(SATA) and provide hints as to the contents of the event. In this case the command is 176 (SMART) with a FIS type of 39 (FIS Register Host to Device). It is important to note that TraceToJson does minimal parsing of the eventData. This is intentional as TraceToJson simply shifts the trace from a proprietary format into a format which only requires a JSON parser to understand.

\subsection{PyTrace}

PyTrace is the Python library responsible for providing programmatic access to the JSON traces generated by TraceToJson. Any subsequent analysis tools (including the Sequential Stream Detector) are expected to be built upon PyTrace. To facilitate this PyTrace provides a reader for consuming the traces created by TraceToJson, a SATA Command Parser for parsing the trace events into logical SATA commands, a Command Statistics Generator for filtering information from a series of commands, and a database access layer. PyTrace itself is written as a series of Python generators to minimize the memory footprint of processing traces containing hundreds of thousands or even millions of events.

\subsubsection{JSONReader}

The JSONReader consumes the JSON trace file produced by TraceToJson and produces events, without buffering, as a Python generator.

\subsubsection{SATA Command Parser}

At its heart the SATA Command Parser is a primitive SATA protocol engine. A trace contains SATA protocol event data as it passes over the wire but such 
events, as individuals, don't represent a complete read or write. An actual read or write, as with other packeted protocols, consists of a series of events (packets) which when taken as a whole constitute the full read or full write. As an example, a successful queued read or write consists of three events: a request (FIS Register Host to Device), an acknowledgment (FIS Device Bits), and a response (FIS Register Device to Host). It is the job of the SATA Command Parser to take each event, parse the eventData array, and group the events per the SATA protocol into logical read and write commands.

SATA uses a FIS (Frame Information Structure) packet to encapsulate and transport the actual SATA command data[11]. Parsing a SATA command therefore requires two phases: first parse the FIS packet to identify the SATA command and then parse the encapsulated SATA command. The difficulty in this parsing lies in how each SATA command uses the FIS packet to store its data. For example, both WRITE_FPDMA_QUEUED and WRITE_DMA use a Host to Device Register FIS (FIS_REG_H2D) packet but WRITE_DMA sets aside a sector count of 0 to actually be 256 sectors while WRITE_FPDMA_QUEUED does not[20]. Slight or major differences such as these requires custom parsing logic for each SATA command or family of commands despite the common FIS packet structure.

The SATA Command Parser is implemented as a Python generator which consumes events, determines the FIS packet type and SATA command type, looks up and calls a parse handler based on the SATA command type, and emits logical commands consisting of several parsed events. The parser does not currently support the full SATA command set and will fail with an assertion error if an unknown command is encountered. It is expected that additional command support will be added as needed from future trace analysis. 


\subsubsection{Command Statistics Generator}

For the analysis work in this thesis the fully parsed SATA commands are of little use. Instead the necessary information (LBA, completion time, etc) must be extracted or computed from individual commands and series of commands. This sort of data filtering is the job of the Command Statistics Generator which extracts or computes data from each of the SATA commands based on a user defined set of fields. The current set of provided fields include:

ID Command ID

Start Time Command Start Time

End Time Command End Time

LBA Logical Block Address

FUA Force Unit Access

Length Sector Count

InterCmdTime Inter Command Time

CCT Command Completion Time

qCCT Queued Command Completion Time

CommandType Command Type (R/W/-)

qDepth Queue Depth

eqDepth Exit Queue Depth

Stream Stream ID

Each field is a functor which computes or extracts a value from the tuple of the previous command, the current command, and the next command. New fields can be easily provided by simply creating a new functor which computes the desired value and passing the new field along with the existing fields. 
The design of the Command Statistics Generator output and the chosen fields are based on a closed source analysis tool provided by Western Digital. The Western Digital tool is capable of generating a CSV file containing the above fields as well as others. The main difference between the approach of the Western Digital tool and the Command Statistics Generator is one of file based data access (Western Digital) versus programmatic data access (Command Statistics Generator). The Command Statistics Generator can reproduce the CSV output of the Western Digital tool.

\subsubsection{Memory Usage}

Table 3.1 shows the memory usage for PyTrace running on the Python 3.2.3 interpreter. Due to the generator based design, this memory usage is consistent regardless of the size of the trace being processed. Python generators act as iterators which create values on the fly rather than traditional buffering based iterators. PyTrace uses mmap to efficiently read the trace file and as a result the host system will show a far greater memory footprint than Table 3.1. 


\begin{tabular}{|r|r|r|}
\hline types & \# objects & total size \\
\hline <class 'str & 9821 & $2.45 \mathrm{MB}$ \\
\hline <class 'dict & 925 & $1.14 \mathrm{MB}$ \\
\hline <class 'type & 435 & $353.44 \mathrm{~KB}$ \\
\hline$<$ class 'code & 2509 & $333.23 \mathrm{~KB}$ \\
\hline$<$ class 'tuple & 1010 & $74.88 \mathrm{~KB}$ \\
\hline$<$ class 'wrapper_descriptor & 912 & $71.25 \mathrm{~KB}$ \\
\hline$<$ class 'weakref & 829 & $71.24 \mathrm{~KB}$ \\
\hline$<$ class 'list & 505 & $66.73 \mathrm{~KB}$ \\
\hline$<$ class 'set & 218 & $54.89 \mathrm{~KB}$ \\
\hline$<$ class 'builtin_function_or_method & 714 & $50.20 \mathrm{~KB}$ \\
\hline$<$ class 'method_descriptor & 612 & $43.03 \mathrm{~KB}$ \\
\hline$<$ class 'getset_descriptor & 385 & $27.07 \mathrm{~KB}$ \\
\hline$<$ class 'abc.ABCMeta & 33 & $26.81 \mathrm{~KB}$ \\
\hline function (__init_-) & 201 & $26.70 \mathrm{~KB}$ \\
\hline <class 'collections.OrderedDict & 2 & $21.32 \mathrm{~KB}$ \\
\hline \multicolumn{2}{|c|}{}
\end{tabular}

Table 3.1: Memory Usage

\subsection{Database}

The final piece of the trace analysis platform is the database storage layer. PyTrace provides powerful, programmatic access to the trace data and is written as a series of generators to reduce memory usage. The downside to being written as a series of generators is that PyTrace inherently consumes a trace from disk and produces a value or series of values in a one-shot process. A single event is read from an input file, parsed and grouped into a logical SATA command, and finally any relevant data is extracted per the field set given to the Command Statistics Generator. Revisiting processed commands requires reprocessing the entire input file a second time or explicitly buffering intermediate values during the initial processing. By storing the trace events, parsed commands, and computed command statistics in a database we alleviate the need to reprocess the same trace repeatedly. An added benefit of database storage is that lightweight visualization and analysis tools can be written to use the database directly with- 
out requiring PyTrace.

There was no shortage of databases to chose from but from a high level the choices can be broken down into relational and NoSQL databases. Relational databases such as MySQL, Oracle, and MSSQL group related data into tables with fixed schemas allowing multiple tables to be joined together by matching fields between tables. NoSQL databases such as CouchDB and MongoDB are non-relational databases that store data as objects or items with no fixed schema. For example, within the same table one object may have two fields startTime and endTime while another object only has one field $i d$. In general one can consider relational databases as having powerful query capabilities while NoSQL databases excel at storing and retrieving large amounts of data with less powerful query capabilities.

In the end MongoDB was chosen for the flexibility of a NoSQL, "schemaless" database and for the fact that MongoDB stores data as JSON-style documents. Since SATA commands are non-uniform in the number of fields they contain trying to store all parsed commands within a single table schema would quickly prove cumbersome as each row in the database would only use a subset of the data columns present. An alternative could be to create a command table (schema: id, type) and a command data table (schema: id, field, value, commandID) to prevent having many unused columns in the command table. Either solution is overly complicated when a NoSQL database allows for items to be stored with varying number of fields. The other reason for choosing MongoDB is a matter of uniformity in using JSON as the representation format. Given TraceToJson already transforms the trace events into a series of JSON objects it is a natural next step to take those same JSON objects and store them in MongoDB.

Currently all database interactions occur through PyTrace using PyMongo. A 
given trace Trace will have three tables created for it: Trace_events, Trace_commands, and Trace_stats. The events table stores the raw JSON events objects produced by TraceToJson. The commands table stores the parsed commands produced by the SATA Command Parser. The stats table stores the command statistics produced by the Command Statistics Generator using all the default fields. This database layout allows for the caching of the results from any component of PyTrace (TraceToJson, SATA Parser, Command Statistics Generator). In this way subsequent analysis of a trace can simply read the data of interest from the closest data set in the chain. 


\section{Chapter 4}

\section{Sequential Stream Detection}

\subsection{The Case for Observing Sequential Streams}

Sequential access is a natural point of interest for magnetic hard drives. It is one of the rare cases where one can receive not only better performance but better reliability as well. Within a single platter, magnetic hard drives store data on a series of concentric circles known as tracks. Sequential operations remain within a single track or small set of tracks with few seeks necessary. Since seeks represent overhead and cause wear on the drive by reducing seeks through sequential access there is an increase in performance (less overhead) and higher reliability (reduced wear).

Most systems force a trade-off between performance and reliability but in the case of magnetic hard drives sequential access gives both at the expense of a more rigid usage pattern. Sequential access even tends to identify related data as file systems and applications try to organize data in sequential segments to leverage the higher performance and increased reliability to be had. As a result 
from a magnetic drive's perspective an ideal world would have a single application storing and retrieving data entirely in sequential segments.

Of course this is not an ideal world. Not all workloads can be broken up into clean, sequential units of IO and even if they could a computer may be running many such sequential application at the same time. Contention between these applications leads to an interleaving of short, sequential segments which when observed from the drive's perspective appears to be non-sequential. From an overhead perspective, Figure 4.1 shows that the two best case scenarios are to only have one sequential steam or have multiple streams where one is allowed to run to completion before commands for the second stream are issued. Allowing the commands to interleave forces the disk to waste time seeking between the different regions. Despite the inefficiency, modern operating systems perform such interleaving in an attempt to allow fair usage of hardware between running processes. 


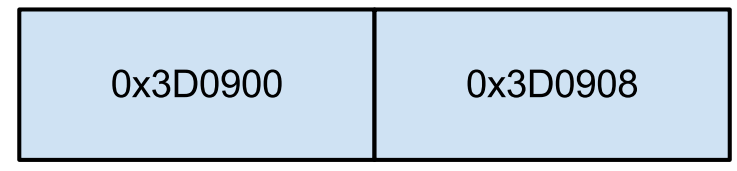

Single Stream

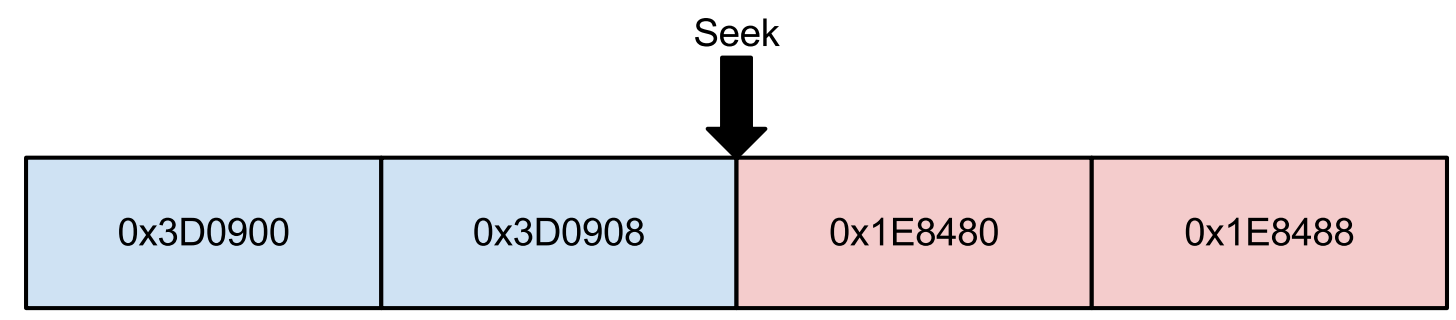

Multiple Streams, Discrete

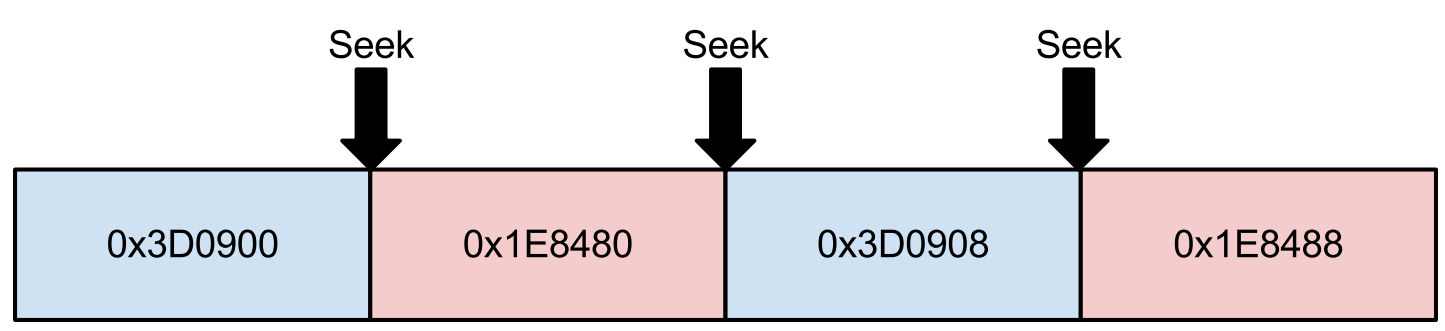

Multiple Streams, Interleaved

Figure 4.1: Disk Access Scenarios

\subsection{Formal Definition of a Sequential Stream}

A pure sequential stream is defined as follows

$$
\begin{aligned}
\forall \beta_{x} \in\left\{\alpha_{0}, \alpha_{1}, \alpha_{2}, \ldots, \alpha_{n}\right\} & :\left(\operatorname{LBA}\left(\beta_{x}\right)+\operatorname{Sector} \operatorname{Count}\left(\beta_{x}\right)=\operatorname{LBA}\left(\beta_{x+1}\right)\right) \\
& \wedge\left(\operatorname{StartTime}\left(\beta_{x+1}\right)>\operatorname{EndTime}\left(\beta_{x}\right)\right) \\
& \wedge\left(\operatorname{StartTime}\left(\beta_{x+1}\right)-\operatorname{EndTime}\left(\beta_{x}\right) \leq \tau\right)
\end{aligned}
$$

where $\alpha$ is a parsed SATA command and $\tau$ is the window size. In other words, a sequential stream consists of a series of commands each of which begins on the 
LBA that the previous command left off on and whose start time is no more than the window size away from the end time of the previous command.

Notice the definition makes no assertion as to the types of command present in the stream (read or write). One might expect a stream to consist entirely of reads or writes but it is not difficult imagine a workload which reads data from the disk, performs some computation, and writes the result to disk following the read data. Restricting streams to be entirely reads or writes would split this workload into two different streams.

\subsection{Window Size}

Window size is the most important aspect of the algorithm given it controls the range over which commands can be considered sequential. The smaller the window size the less likely it is unrelated commands will be grouped as sequential at the expense of missing commands which are related but too far apart in time. Figures 4.2 and 4.3 show the affect of window size on the streams detected on a trace generated from mplayer playing back a video file. Each plots the lifespan of a stream as a function of time with the only difference being the window size used. The $\mathrm{y}$ axis is the stream id while the $\mathrm{x}$ axis is time; such a graph shows the lifespan of streams in time. There is no locality relationship between stream ids only within a single stream id per the invariant defined in 4.2. Figure 4.2 uses a window size of 1 second which leads to a graph with many short streams of at most a couple of seconds. Figure 4.3 uses a window size of 10 seconds which collapses most of the initial streams into a single stream. It is easy to see from these two graphs how necessary choosing the correct window size is from an analysis perspective. 


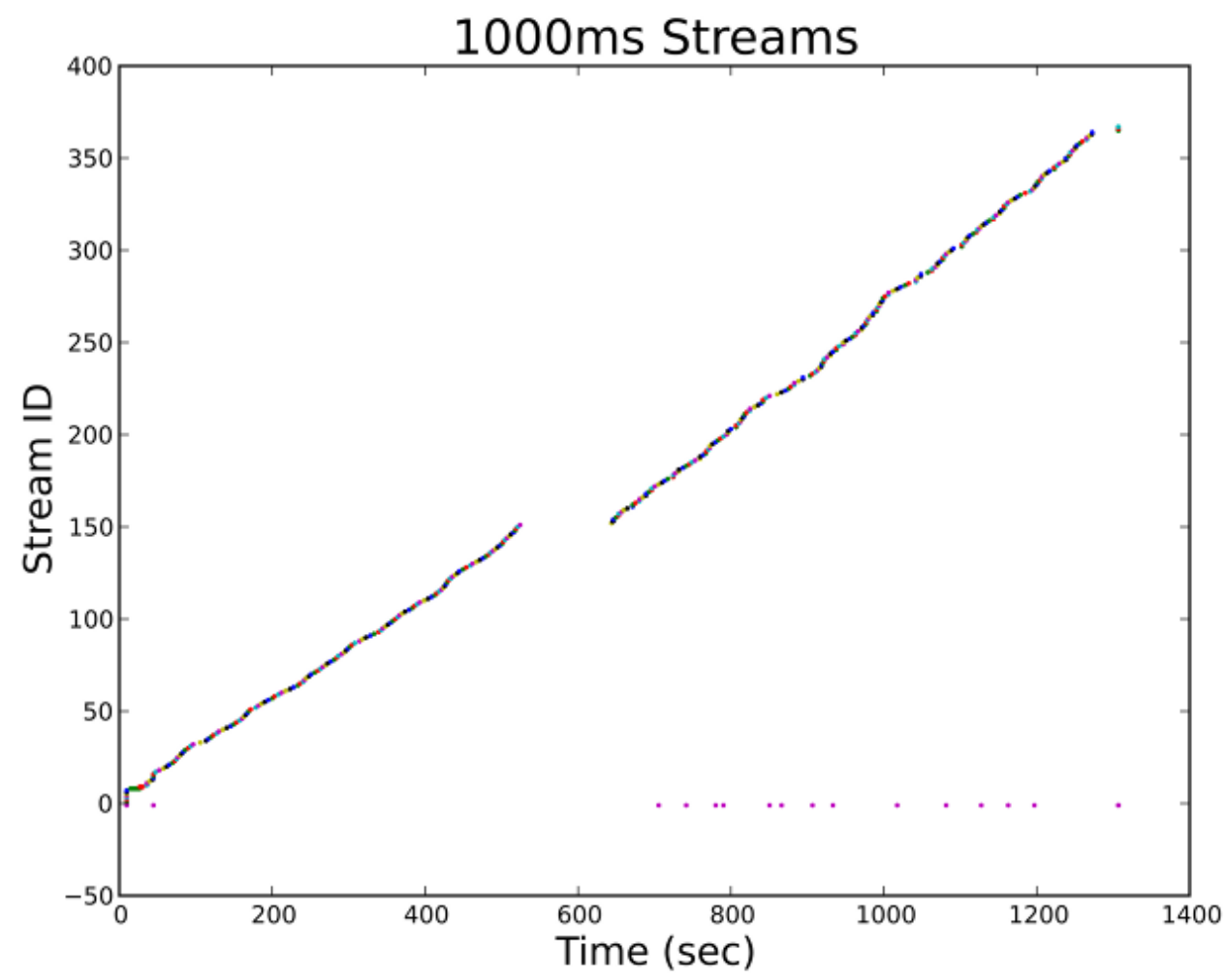

Figure 4.2: mplayer video playback on Btrfs. Window size 1s 


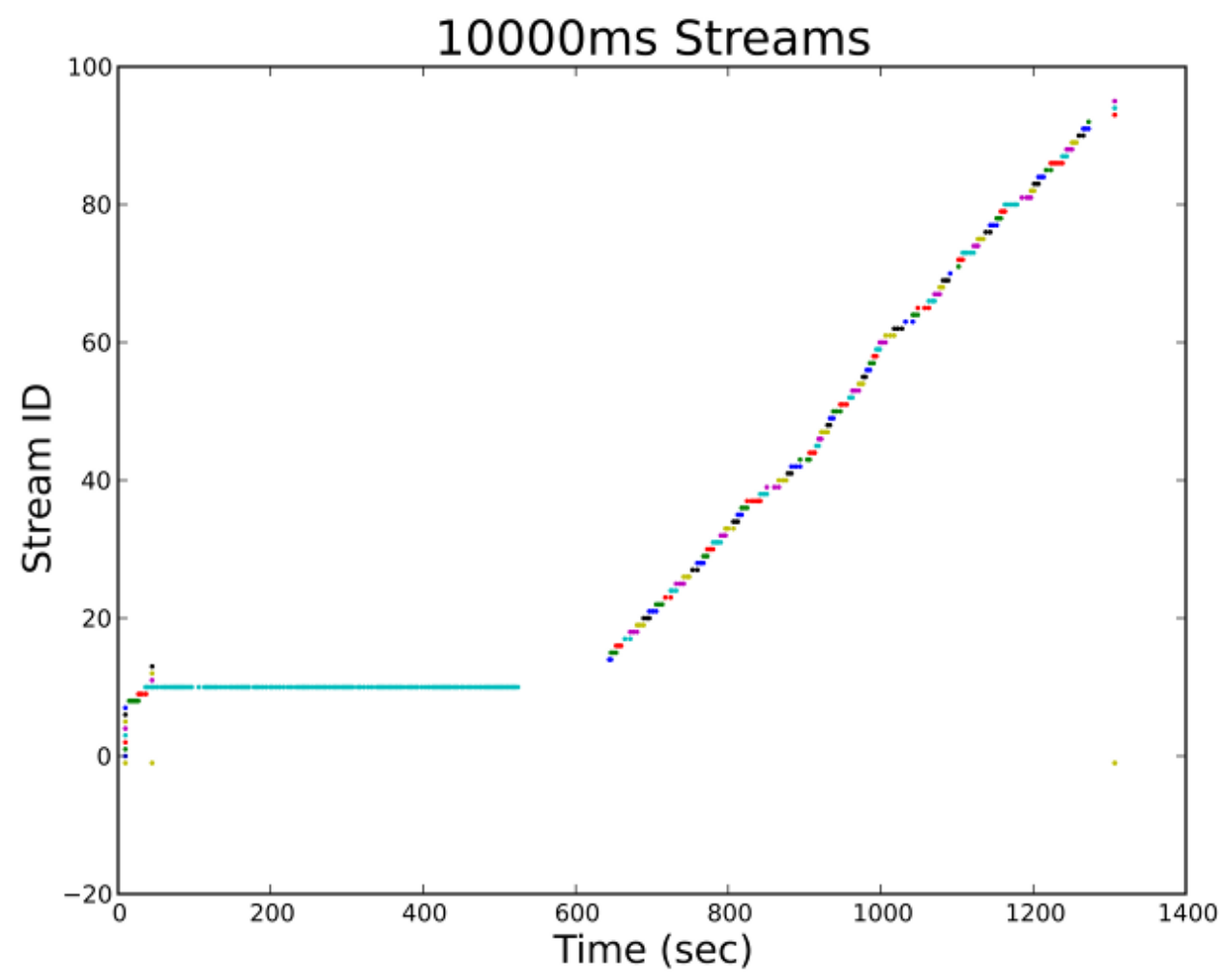

Figure 4.3: mplayer video playback on Btrfs. Window size 10s 
Figure 4.4 shows LBA as a function of time for a synthetic workload consisting of two sequential streams (a read and a write) with random IO mixed in (random reads, random writes, and mixed random reads and writes). Reads are represented as blue points while writes are represented as red points. The sequential read takes place between LBA 0 and 2000000 while the sequential write is between 2000000 and 4000000; all other IO is random. Figure 4.5 demonstrates that with a window size of 10 seconds we can identify the read and write stream (blue and purple horizontal lines). Actually, the write is split over two streams where the first stream is allocated right next to the read stream and as a result doesn't appear clearly in the graph. This split is due to the large gap between 30 and 50 seconds. Due to the relatively large window size otherwise random commands are joined into short (short in number of commands), disjoint streams which can be seen all throughout the graph. 


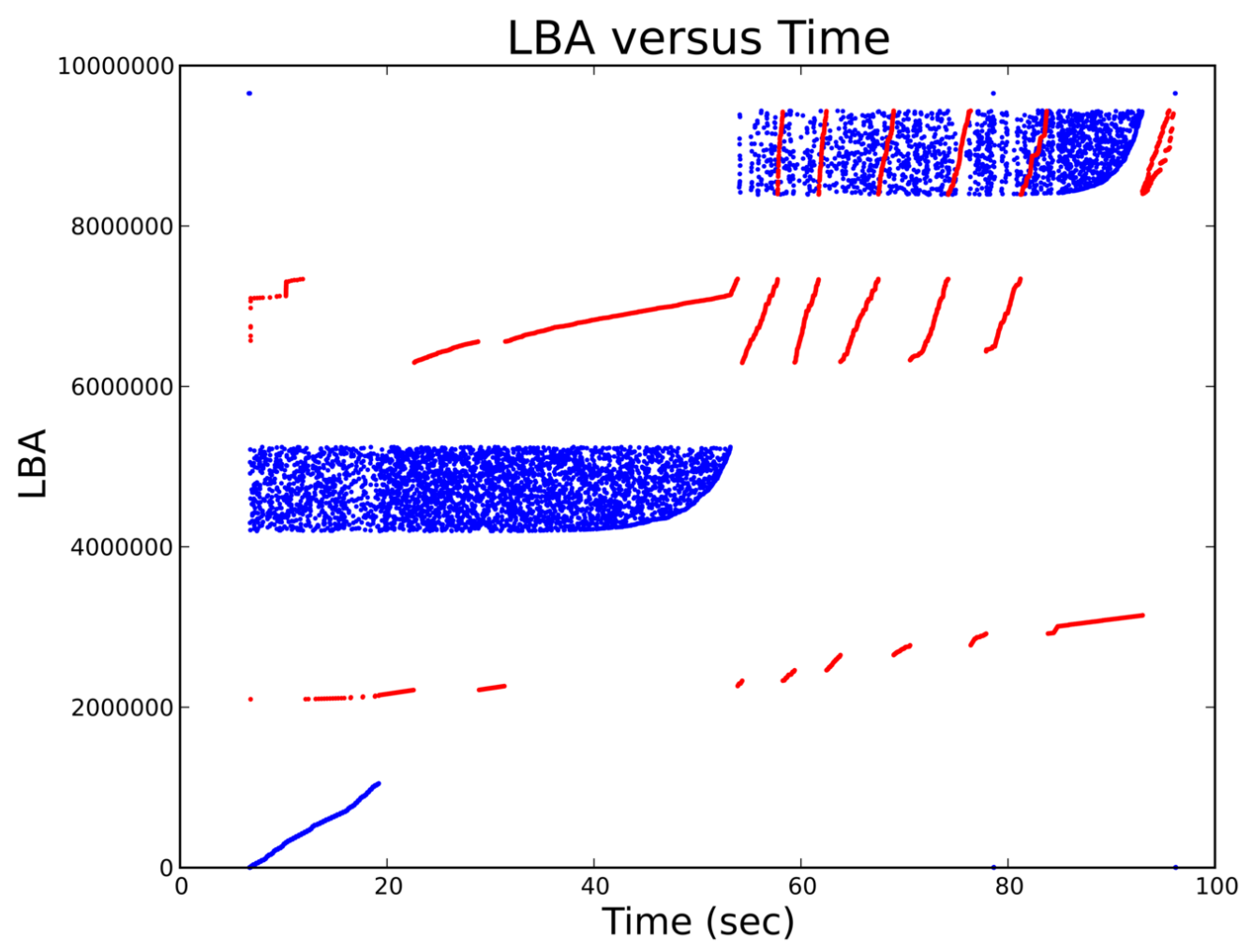

Figure 4.4: fio mixed sequential and random on the raw block device 


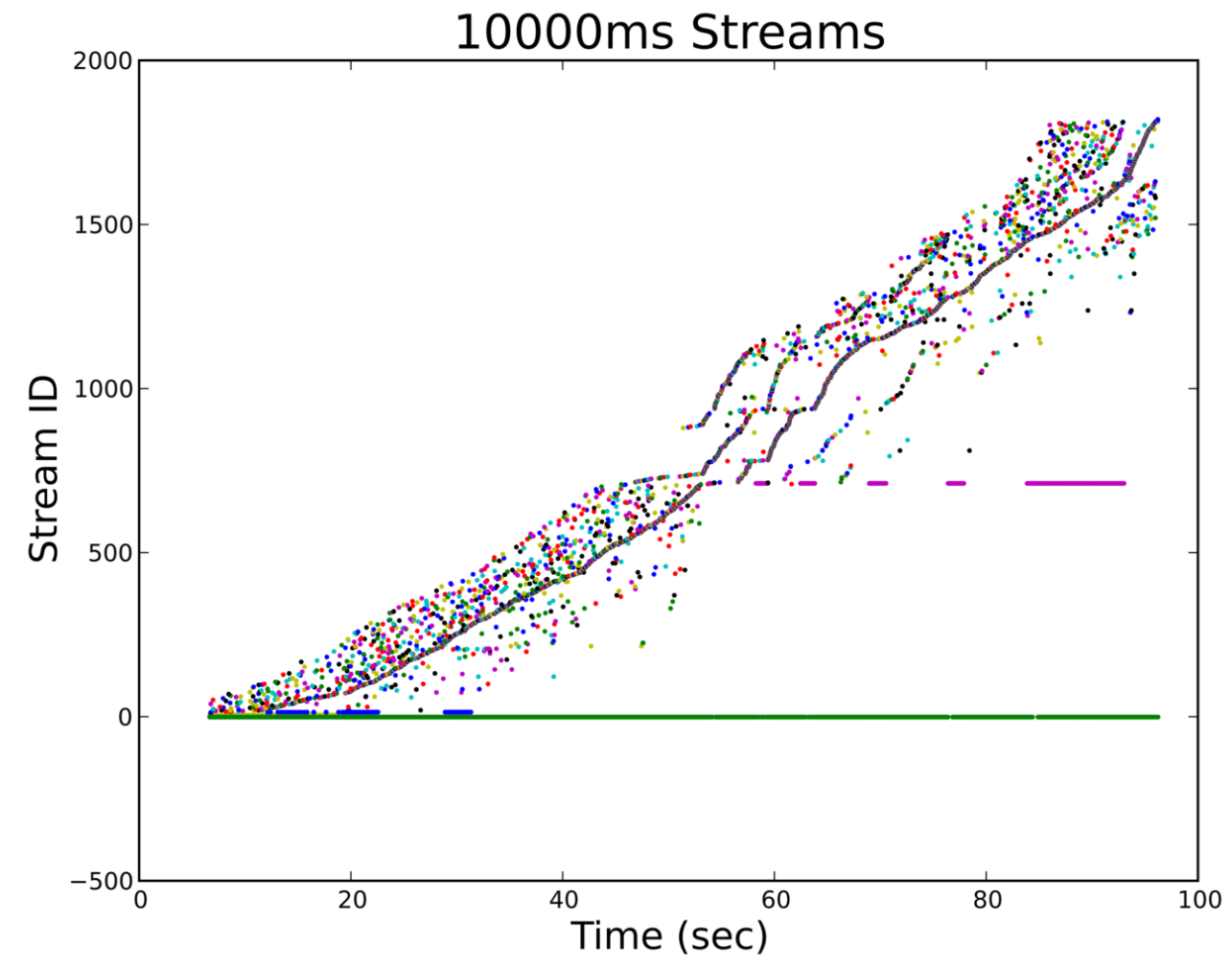

Figure 4.5: fio mixed sequential and random on the raw block device. Window size 10s 
The mplayer (Figures 4.2 and 4.3) and fio (Figure 4.5) traces demonstrate the importance of correct window size selection. A window size that is too small will split a single stream into many smaller streams when the gaps between commands exceed the window size. On the other hand if the window size is too large the noise in the graph will increase as otherwise independent commands are incorrectly grouped together into long and disjoint streams.

\subsection{A Sequential Stream Detection Algorithm}

The sequential stream detection algorithm is a sliding window over the trace where the size of the window is set to $\tau$ as defined in 4.2. Each time a new command is encountered any commands currently in the window with an end time that is $\tau$ or more away from the new command are evicted from the window and no longer considered for any streams. The window is a chronologically ordered list so when scanning from the beginning of the window the first non-expired command encountered signals the end of expired commands contained in the window. If a command is evicted without being assigned to a stream it is assigned to the invalid stream (Stream ID -1). After eviction the new command is matched against the first command within the window (from oldest to newest) that it is found to be sequential to. If the earlier command is already bound to a stream the new command is assigned to the same stream and the earlier command is evicted from the window. If the earlier command is part of no stream a new stream is allocated and both commands are assigned to the stream before the earlier command is evicted. Finally, if no command can be found that the new command is sequential to then the new command is placed within the window assigned to no stream as a potential match for future commands. This algorithm 


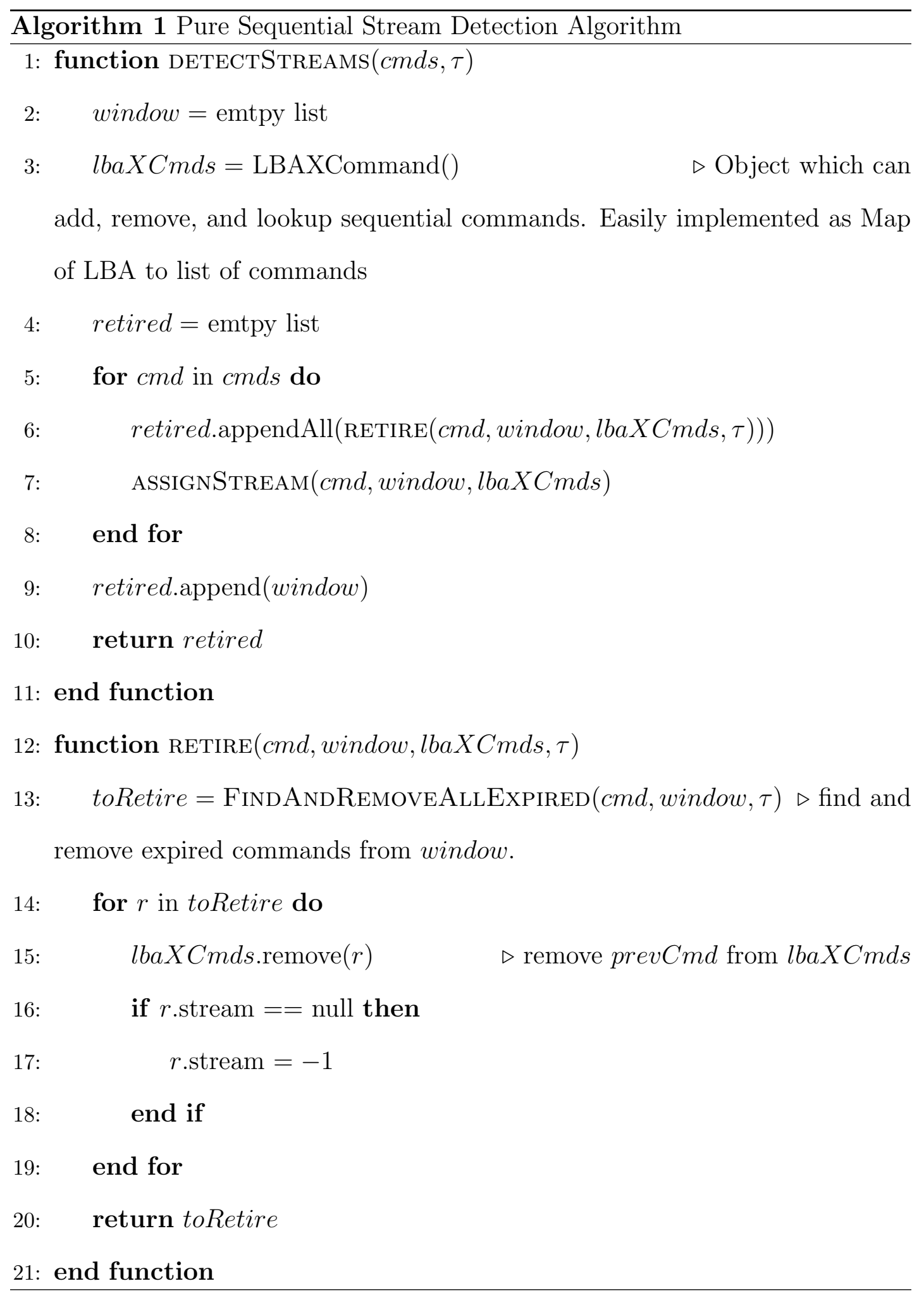


22: function ASSignStream $(c m d$, window, lbaXCmds)

23: $\quad$ prevCmd $=l b a X C m d s \cdot \operatorname{lookup}(\mathrm{cmd}) \quad \triangleright$ lookup oldest command $\mathrm{cmd}$ is sequential to

24: $\quad$ if prevCmd $\neq$ null then

25: $\quad$ if prevCmd.stream $==$ null then

26: $\quad$ prevCmd.stream $=$ NextSTREAmID(void) $\quad \triangleright-1$ is the Invalid Stream

27: $\quad$ end if

28: $\quad$ cmd.stream $=$ prevCmd.stream

29: $\quad$ lbaXCmds.remove $($ prev $C m d) \quad \triangleright$ remove prevCmd from lbaXCmds

30: $\quad$ end if

31: $\quad$ lbaXCmds.add $(\mathrm{cmd}) \quad \quad \quad$ add $\mathrm{cmd}$ to lbaXCmds

32: $\quad$ window.append $(\mathrm{cmd})$

33: end function 
makes no distinction between read and write commands. A stream consisting of alternating reads and writes would receive a single stream id as long as the reads and writes were in fact sequential.

The run-time complexity of the algorithm is $\mathrm{O}(n)$ where $n$ is the number of commands. The worst case scenario occurs when setting the window size to the duration of the entire trace with no streams present. In this situation

all commands will be added and kept within the window. Performance would degrade in this situation but because the window is a sorted list it is not necessary to search through the entire list looking for expired commands. When searching through the window from beginning to end, once a non-expired command is encountered one knows that there are no other expired commands further in the list since all subsequent commands are newer. So when the window size is set to the duration of the trace the first command in the window will never expire due to the window size and no further searching is necessary. Performance therefore remains $\mathrm{O}(n)$ in the worst case.

\subsection{Implementation}

The sequential stream detection is implemented in Python on top of PyTrace; situating itself between the SATA Command Parser and the Command Statistics Generator (Figure 4.6). The Detector consumes the commands emitted by the SATA Command Parser and emits the same commands in received order with the addition of a new field on the command object identifying the stream. The overhead added by the Detector is a buffering delay by which the Detector will not emit a command until the command is expired due to stream timeout, another command in the stream is found, or there are no further commands to assign 
streams to.

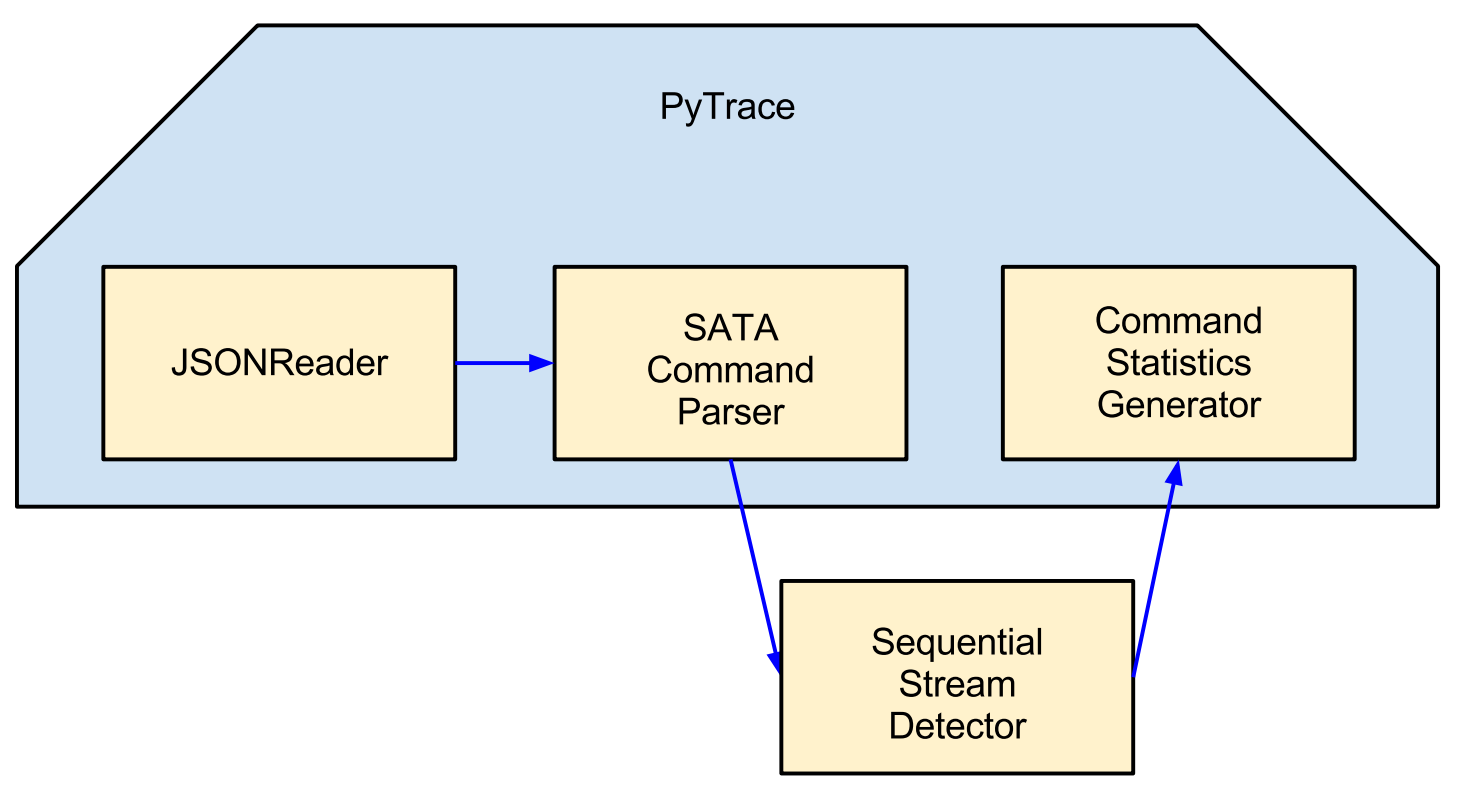

Figure 4.6: Sequential Stream Detector

As with the other PyTrace components the Sequential Stream Detector is implemented as a Python generator to minimize memory footprint. Expired commands are immediately yielded to limit buffering to only the commands alive in the window. A benefit of the Python implementation of the detection algorithm is that with Python's dynamic nature, the field with which the Stream ID is assigned to can be specified at run-time. This allows for chaining together multiple Detectors with different window sizes to observe the differences in stream allocation with varying window size.

\begin{tabular}{|l|l|}
\hline Command Count & Time (sec) \\
\hline 19 & 0.171 \\
\hline 5557 & 13.097 \\
\hline 38298 & 78.786 \\
\hline 1019817 & 619.517 \\
\hline
\end{tabular}

Table 4.1: Sequential Stream Detection Runtimes. Window Size 100ms 


\section{Chapter 5}

\section{Validation}

\subsection{Trace Analysis Platform Validation}

PyTrace was validated by comparing CSV files generated by PyTrace with the CSV files generated by the Western Digital reference tool as described in 3.3.3. PyTrace CSV generation exercises all components of PyTrace save for the database layer which could still be instrumented into the test. There are three known differences between the data produced by PyTrace and the Western Digital tool. First and least important, PyTrace does not pad timestamps to six decimal places. The second difference is that PyTrace makes no attempt to match the command ID assignment used by the Western Digital tool. PyTrace sets the command ID to the ID of the first event in the command while the Western Digital tool creates a new domain of IDs for commands independent of event IDs. Finally, PyTrace filters fewer commands when compared to the Western Digital tool. The only difference with important impact on the values produced by PyTrace is the command filtering. By filtering less commands it is possible that values which are computed by comparing commands may differ 
between PyTrace and the Western Digital tool.

\subsection{Sequential Stream Detection Validation}

The Sequential Stream Detection algorithm was validated against a series of synthetic workloads with a known number of sequential streams. To pass validation the Sequential Stream Detector must correctly identify all known sequential streams within a synthetic workload barring incorrect window size and interference with random IO if random IO is present. The tools used to generate the synthetic workloads were $d d$ (UNIX file copy utility) and fio (hard drive stress test/benchmarking tool), each run against the raw disk with no background IO present.

\subsubsection{Workloads Run}

The following is the list of validation workloads run.

dd - one process

one instance of dd writing sequentially to disk

dd - two processes

two instances of dd writing sequentially to disk

dd - three processes

three instances of dd writing sequentially to disk

dd - four processes

four instances of dd writing sequentially to disk

fio - step threads

following run sequentially: 1 write thread, 2 write threads, 4 write threads, 
1 read thread, 2 read threads, 4 read threads

\section{fio - mixed sequential and random}

following run in parallel: sequential read, sequential write, random read, random write, and mixed random $\mathrm{read} /$ write

\section{fio - pure random}

all random access

\subsubsection{Results}

Table 5.1 summarizes the results of running the Sequential Stream Detection on the validation workloads. \# Seq. is the expected number of sequential streams and \# Detected Seq. is the number of detected sequential streams. It is important to note that the fio test cases assigned substantially more streams than the values listed in the table due to the presence of random IO in some of the tests. To account for this only sequential streams of at least 200 commands in length are considered a "detected" stream.

\begin{tabular}{|l|c|c|c|}
\hline Name & \# Seq. & \# Detected Seq. & Window Size $\tau$ (sec) \\
\hline$d d$ - one process & 1 & 1 & 100 \\
\hline$d d$ - two processes & 2 & 2 & 100 \\
\hline$d d$ - three processes & 3 & 3 & 100 \\
\hline$d d$ - four processes & 4 & 4 & 100 \\
\hline fio - step threads & 14 & 14 & 10 \\
\hline fio - mixed sequential and random & 2 & 2 & 10 \\
\hline fio - pure random & 0 & 0 & 10 \\
\hline
\end{tabular}

Table 5.1: Sequential Stream Detection Validation Results

The window size for $d d$ may seem strangely high and it is in fact the case that 100 seconds is far higher than is necessary. The actual window size required for correct detection is closer to 20 seconds. From the set of window sizes used 
(10ms, 100ms, 1s, 10s, 100s) 10s was slightly too small and while 100s seconds showed the correct number of streams despite being larger than necessary. Given the synthetic nature of the tests themselves it is safe for both the $d d$ and fio tests to have a window size much larger than necessary. This is due to the fact that the synthetic tests had each IO pattern run (sequential read, sequential write, random read, etc) on a distinct region of the disk such that no two patterns overlapped. Had there been overlap it is possible that setting the window size too high would have grouped unrelated commands together in a stream that just happened to meet the criteria for sequentiality.

Table 5.2 shows the number of detected streams for the validation workloads with a window size that is too small. Half the $d d$ workloads show an increase in the number of workloads detected while the other half show a decrease. fio - step threads is the only fio workload to change. An increase in the number of detected streams is expected given the smaller window size. A smaller window size causes streams to be cut off more quickly and subsequent sequential commands which would have been part of the cut off stream are allocated a new stream. The $d d$ workloads which decreased in the number of streams did so due to the 200 command filter. The number of allocated streams increased but with the 200 command filter fewer streams made it over the bar. The number of detected streams did not increase for the last two fio workloads due to the 200 command filter as well. 


\begin{tabular}{|l|c|c|c|}
\hline Name & \# Seq. & \# Detected Seq. & Window Size $\tau$ (sec) \\
\hline$d d$ - one process & 1 & 2 & 10 \\
\hline$d d$ - two processes & 2 & 1 & 10 \\
\hline$d d$ - three processes & 3 & 10 & 10 \\
\hline$d d-$ four processes & 4 & 3 & 10 \\
\hline fio - step threads & 14 & 16 & 1 \\
\hline fio - mixed sequential and random & 2 & 2 & 1 \\
\hline fio - pure random & 0 & 0 & 1 \\
\hline
\end{tabular}

Table 5.2: Sequential Stream Detection Incorrect Window Size 


\section{Chapter 6}

\section{Trace Analysis Using Sequential Stream Detection}

\subsection{Stream Graphs}

Plotting the detected streams as a function of time yields some interesting results. Such a graph demonstrates the lifespan of streams over the course of the entire trace. During some workloads, long prominent streams emerge while other workloads yield only small, short lived streams. Other features such as interleaving between streams become readily apparent in such a graph.

One of the more interesting aspects of a stream graph is its ability to identify randomness vs sequentiality in LBA access. Naturally one is looking for long, horizontal lines which identify prominent streams. Even a trace consisting entirely of small streams conveys information as to the randomness and sequentiality within the slope of the trending line formed by the allocation of streams (left most point of each stream). A large number of stream allocations in a short period of time 
creates a steep line which implies high randomness or discontinuity in LBA access. A more horizontal trending line implies either higher sequentiality or less dense random IO.

Figure 6.1 shows the streams (Window Size: 10s) for a rebuild of the Fedora 16 GCC RPM on Btrfs. There are no prominent streams which stand out on the graph given the long line at the bottom is the invalid stream (Stream ID -1). In fact the longest streams in the graph are a handful between 100 and 200 seconds. The graph does show regions of high randomness compared to sequentiality which are apparent in the slope of the trending line. Each of the dense clustering of blue (high number of random reads) in Figure 6.2 corresponds to a vertical jump in Figure 6.1 at the same time. Such jumps include 3000, 8000, and 9500 seconds. Segments of Figure 6.2 with lower random reads (less blue) show the dominance of the more sequential writes (red lines) with a leveling off of Figure 6.1. Figure 6.3 shows the LBA and Stream Graph superimposed to more clearly see where vertical jumps in the Stream Graph correspond to random access in the LBA graph. 


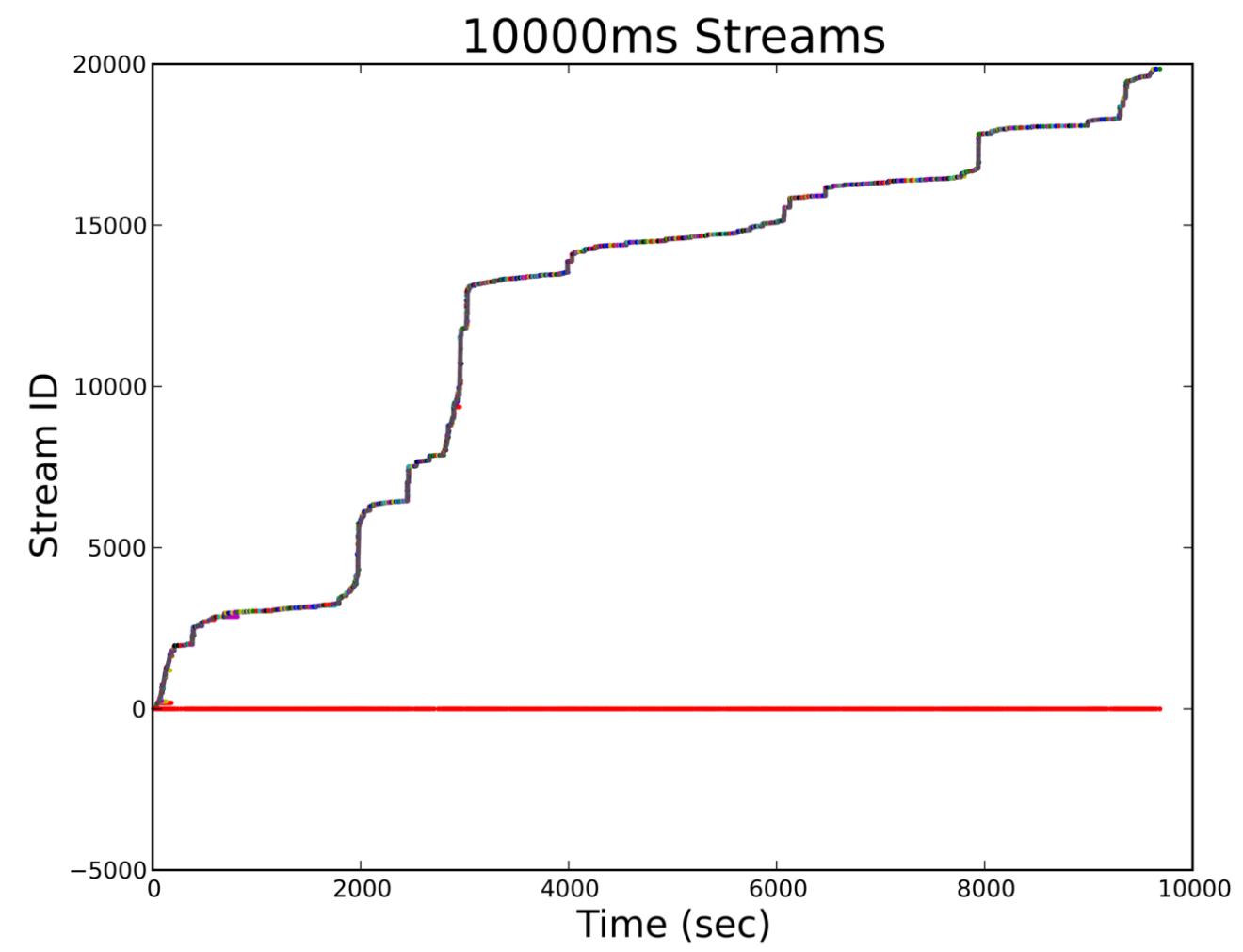

Figure 6.1: Fedora 16 GCC RPM rebuild on Btrfs - Streams 


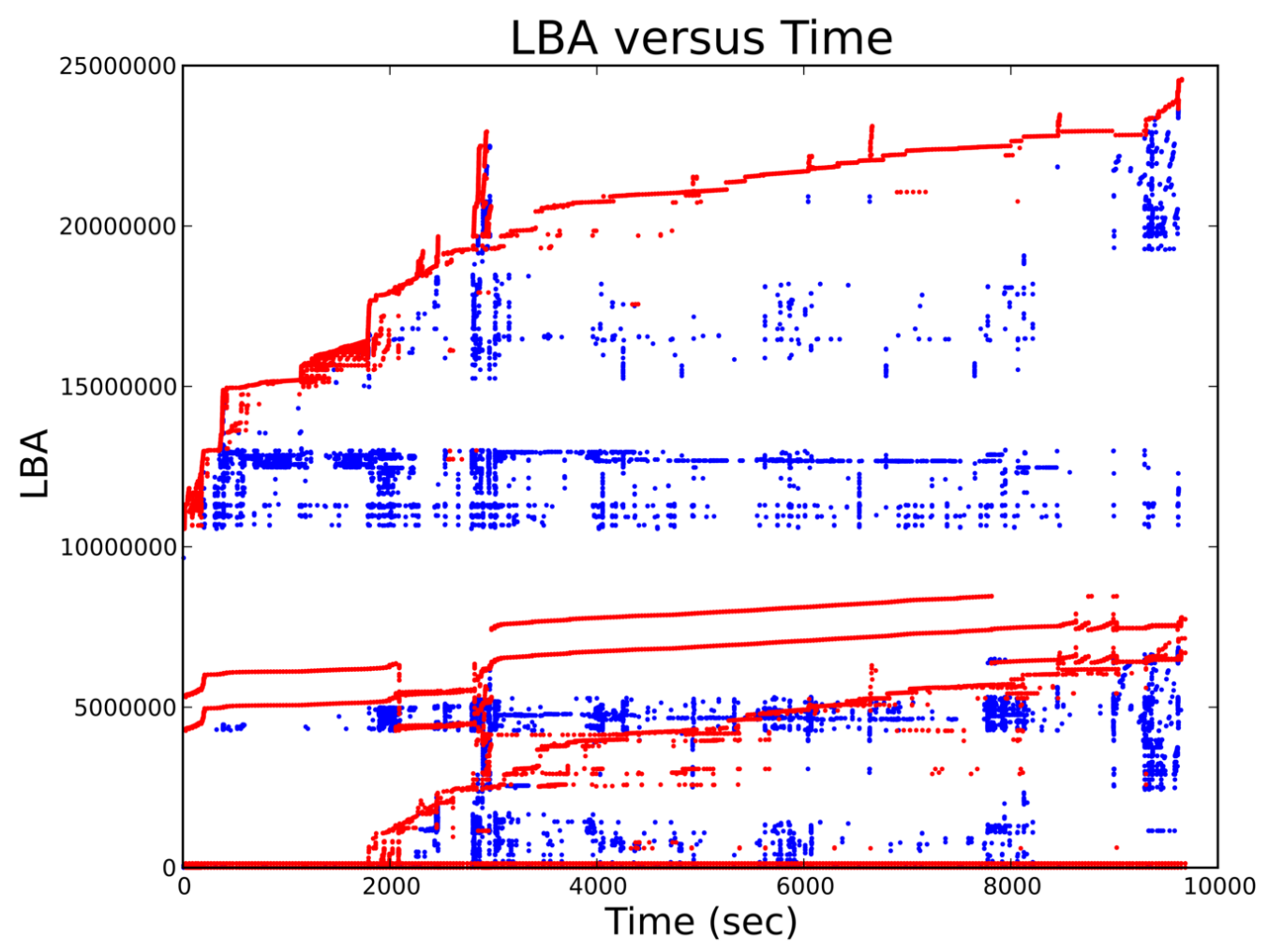

Figure 6.2: Fedora 16 GCC RPM rebuild on Btrfs - LBA 


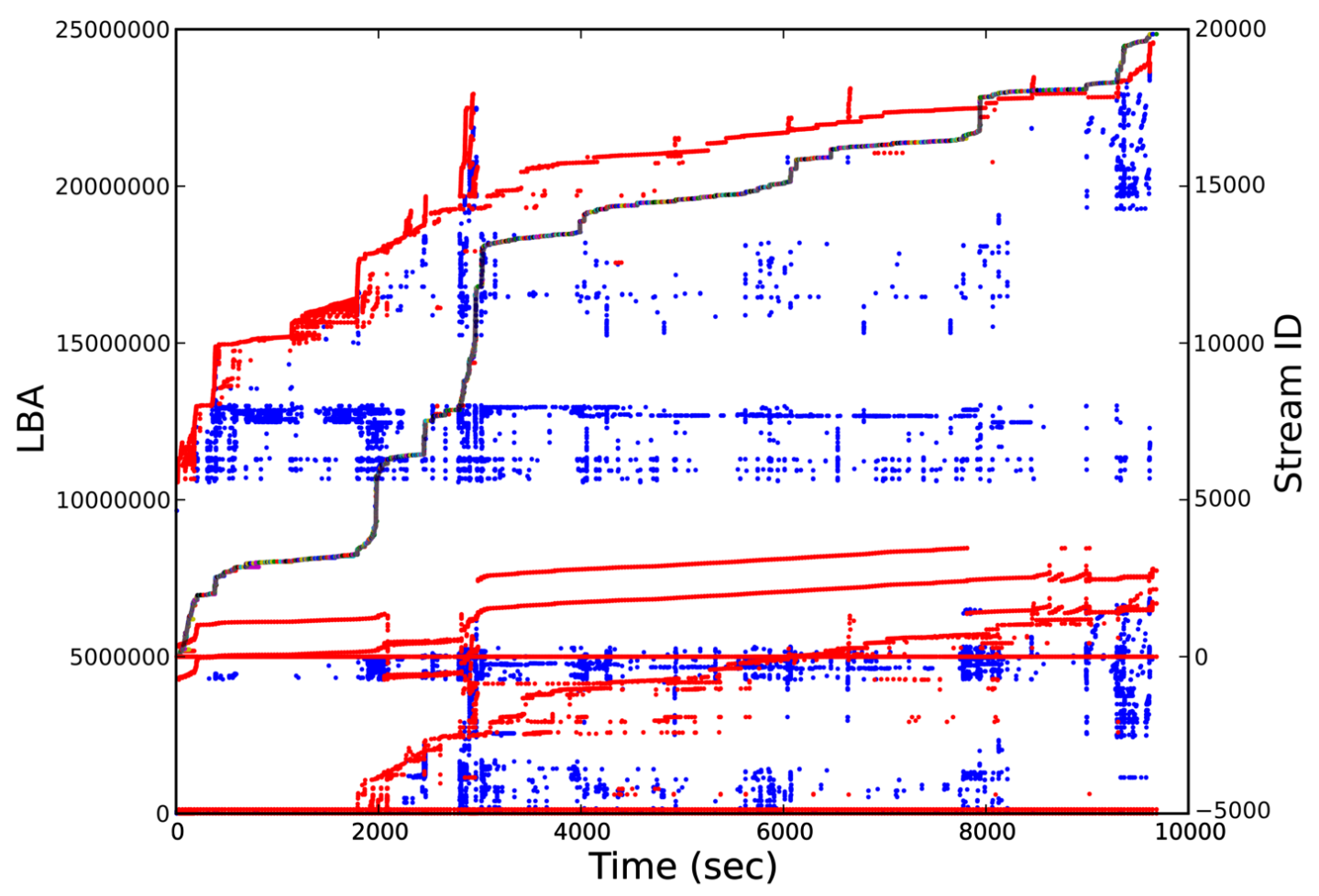

Figure 6.3: Fedora 16 GCC RPM rebuild on Btrfs - LBA and Streams 


\section{$6.2 \mathrm{dd}$}

The $d d$ traces used to validate the stream detector are interesting due to how surprisingly bursty the disk IO is. One would expect $d d$ to write steadily to disk given $d d$ is designed to copy files with transformation. What we see is that even a single instance of $d d$ routinely has 10 or more second gaps between IO chunks (Figure 6.4). There are two possible explanations for this behavior. First, /dev/urandom was the source used for $d d$ to copy from. It is possible /dev/urandom is slow and the long delays were due to the generation of more random data. The other explanation is that $d d$ or the Linux kernel is doing a large amount of buffering before issuing queued commands which are serviced quickly. 


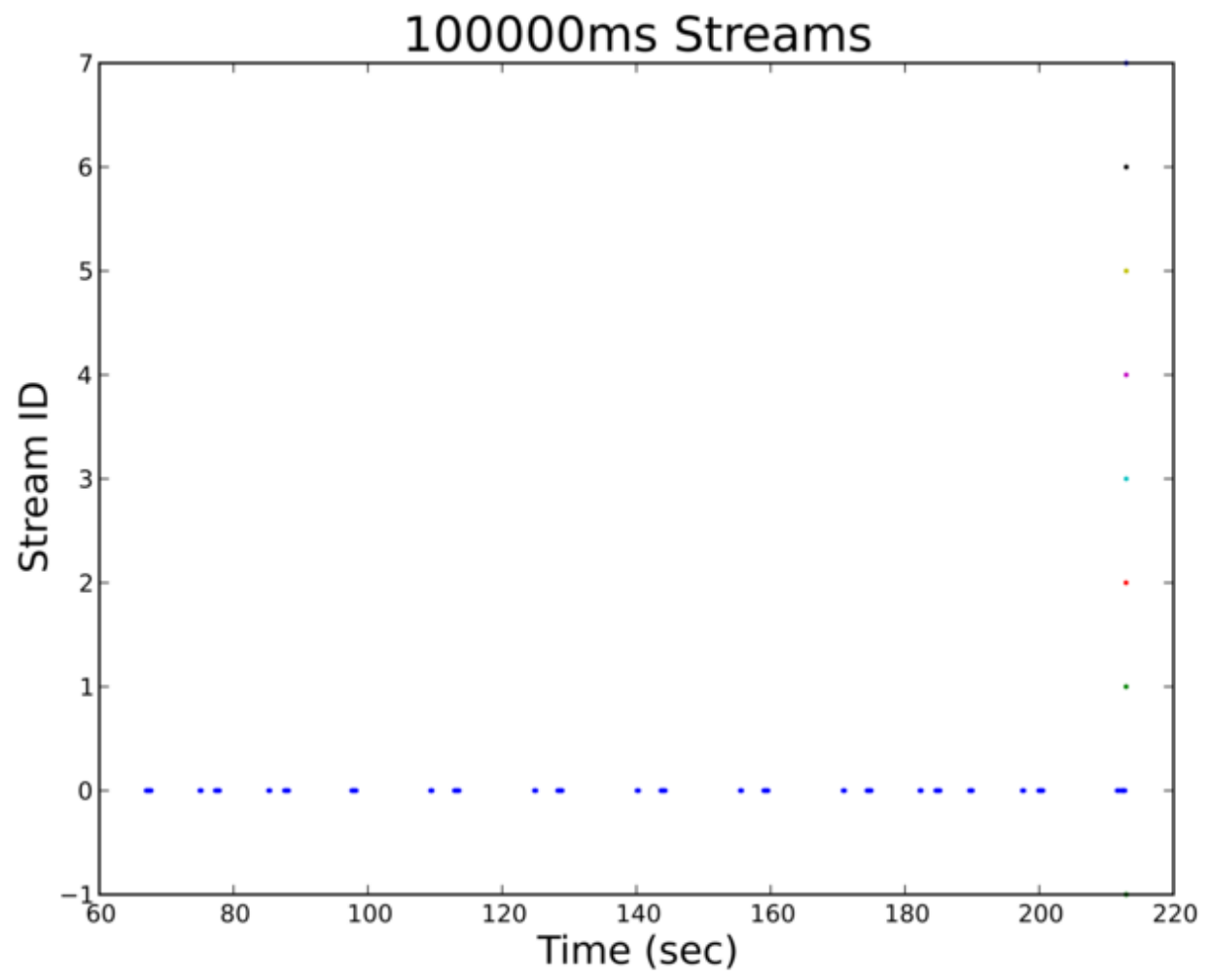

Figure 6.4: $\mathrm{dd}$ if $=/ \mathrm{dev} /$ urandom of $=/ \mathrm{dev} / \mathrm{sdb} 1 \mathrm{bs}=4 \mathrm{~K}$ 


\section{3 mplayer}

Another benefit of the Stream Graph when compared to a graph of LBA as a function of time is the ability of the Stream Graph to quickly show regions as being less sequential than expected. One would expect a trace of mplayer playing back a video file to be a highly sequential operation and therefore have few streams. The LBA graph (Figure 6.5) seems to echo this assumption in the long linear read present in the graph. There is a pause in activity between 600 and 700 seconds but other than the pause the entire read appears to be sequential. In fact, the 600 to 1300 seconds region consists of many relatively small streams (78 streams) which can be seen in the Stream Graph. Figure 6.6 shows the Stream Graph and Figure 6.7 shows the LBA and Stream Graph superimposed. What the LBA graph does not show due to the scale of the graph is that despite the reads sequentially trending upward there are small jumps in LBA accessed which break up the otherwise contiguous sequential read. It is possible to see this in the LBA graph but it requires looking at the correct region of the graph at the correct scale. The Stream Graph readily shows the anomaly (although the graph offers no explanation) regardless of scale. 


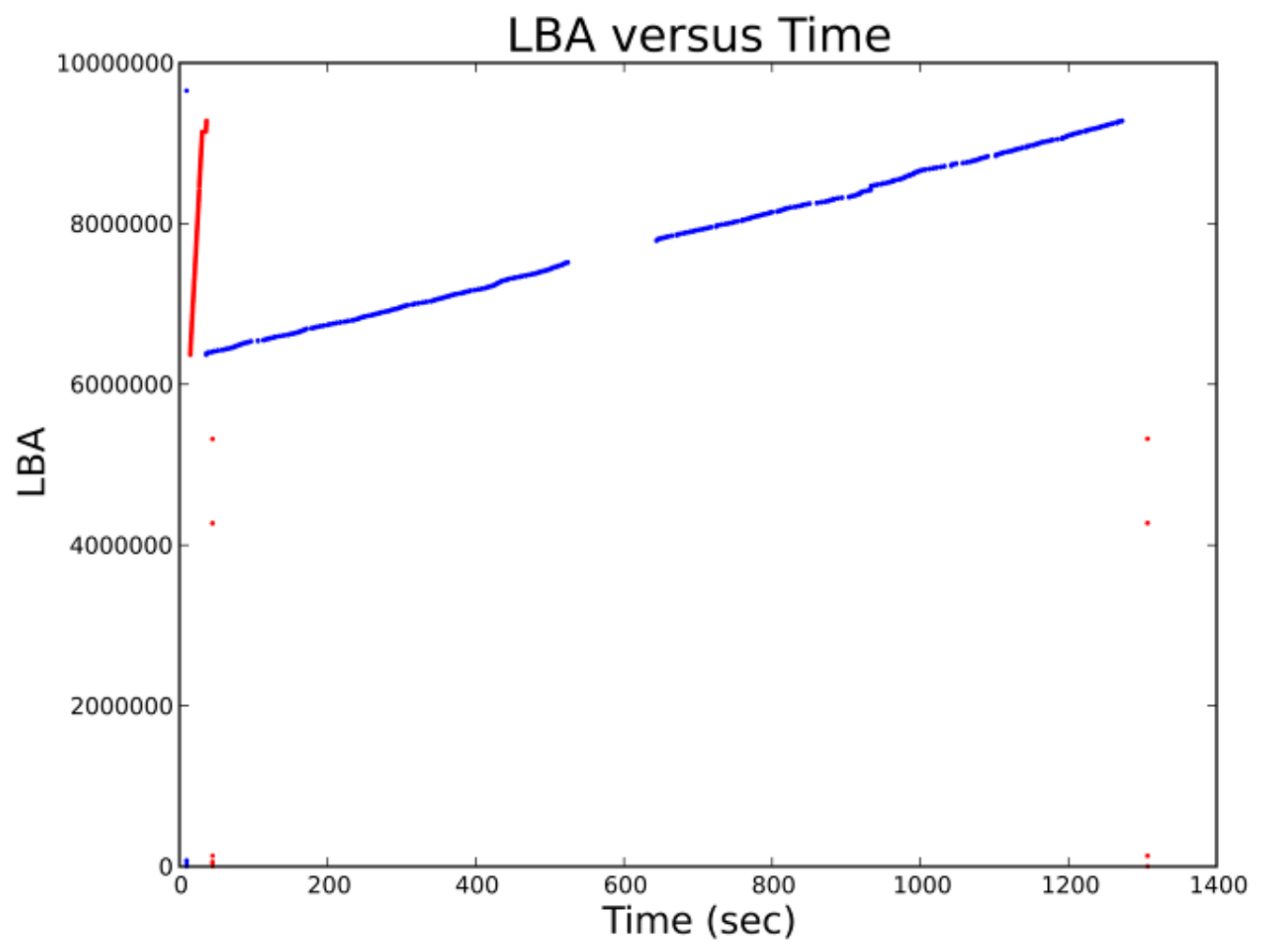

Figure 6.5: mplayer video playback on Btrfs. LBA as a function of time 


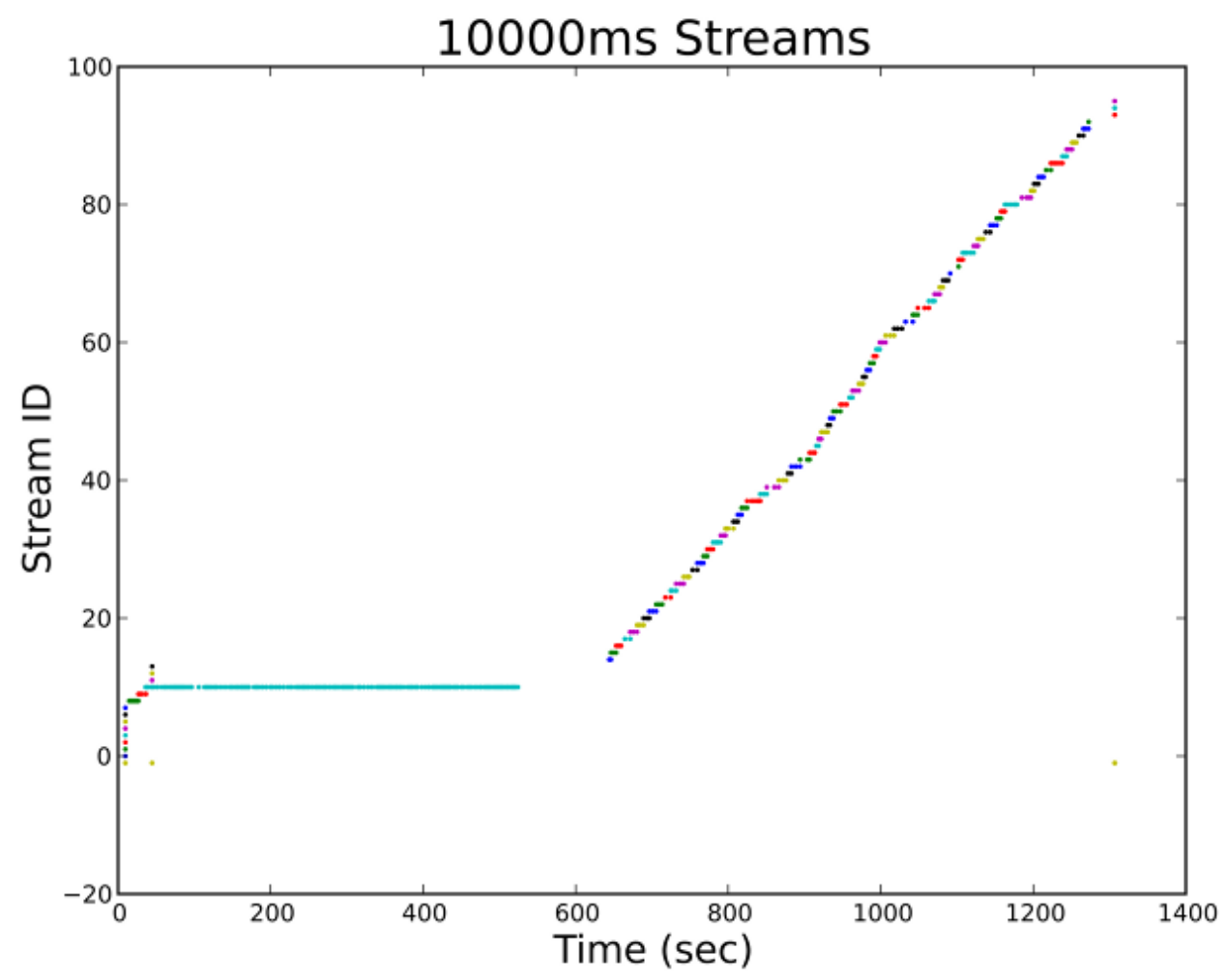

Figure 6.6: mplayer video playback on Btrfs. Window size 10s 


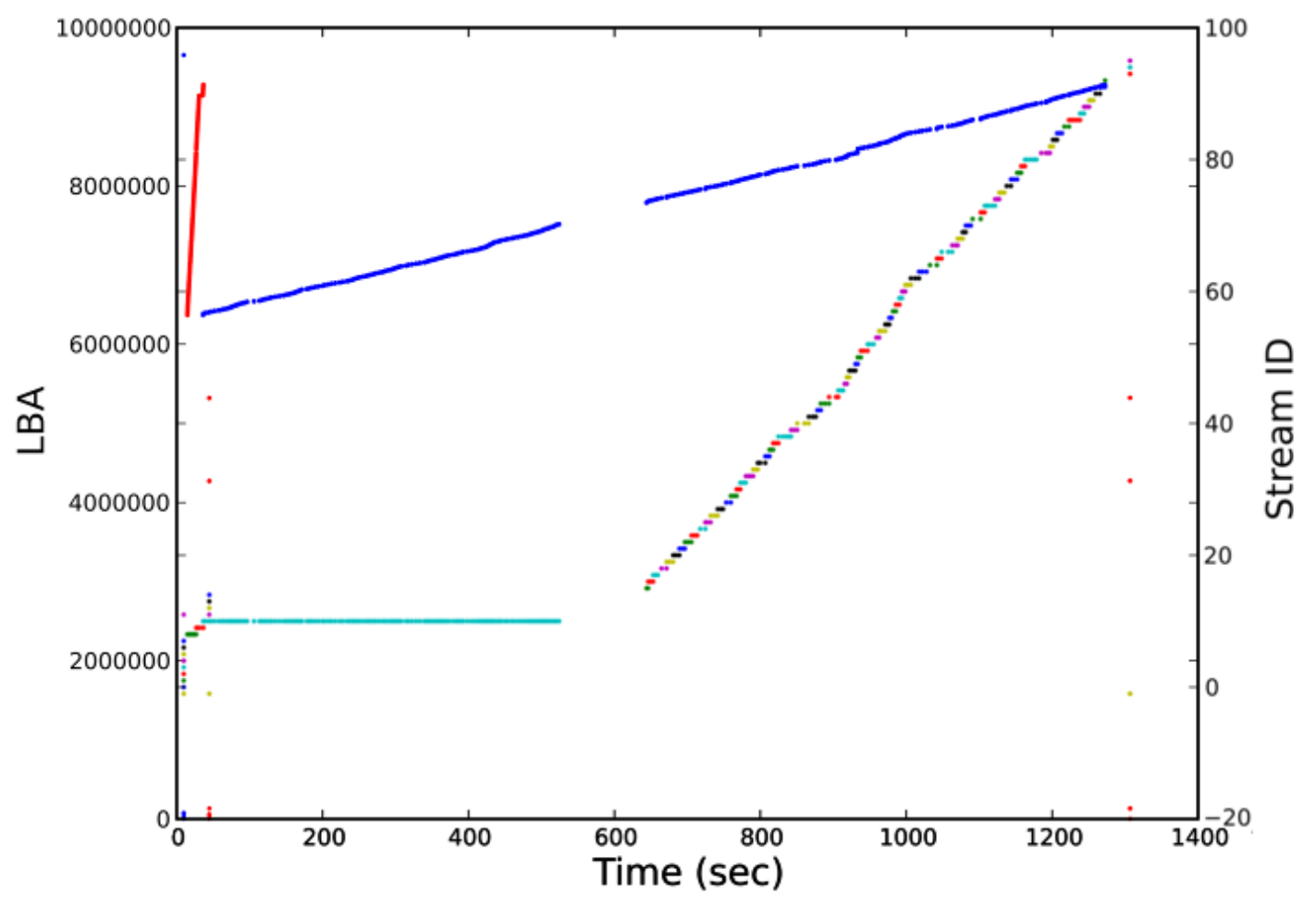

Figure 6.7: mplayer video playback on Btrfs. LBA and Streams 


\subsection{Trace Fingerprinting}

It is important to clarify that the streams shown by the Stream Graph with a large window size at best reveal long term application or OS level sequentiality in disk usage. At worst, the graph correlates completely unrelated commands that happen to fall sequentially per the definition above. In either case there is potentially little meaning to be had from the hard drive's perspective in such a graph with a large window size. The time scales are too long with too many intermediate commands to consider the drive and cache state when a sequential stream was paused and later resumed.

The Stream Graph does serve as a useful tool for identifying and classifying workloads which appears to be consistent across file systems. Given different file systems with different disk usage properties running the same workload on each of the file systems and generating the corresponding Stream Graphs yields noticeably similar graphs. Compare Figure 6.8 to Figures 6.9 and 6.10. The difference between Figure 6.9 and 6.10 is that in Figure 6.10 the LBAs plotted are restricted to those under 25000000. Other than the one difference all three graphs are of the same workload with the former on Btrfs and the latter two on ext2. It seems likely that an untrained eye would be unable to tell whether the workload run on the two file systems was the same or not. Now when we compare Figures 6.1 and 6.11 the similarity between the two graphs is readily apparent despite the difference in the number of streams allocated (20000 compared to 50000). It seems easy to conclude from these two graphs that the two workloads run are indeed the same.

The reason the Stream Graph clearly identifies the workload where the LBA graph struggles is in the Stream Graph's focus on locality and temporality of 


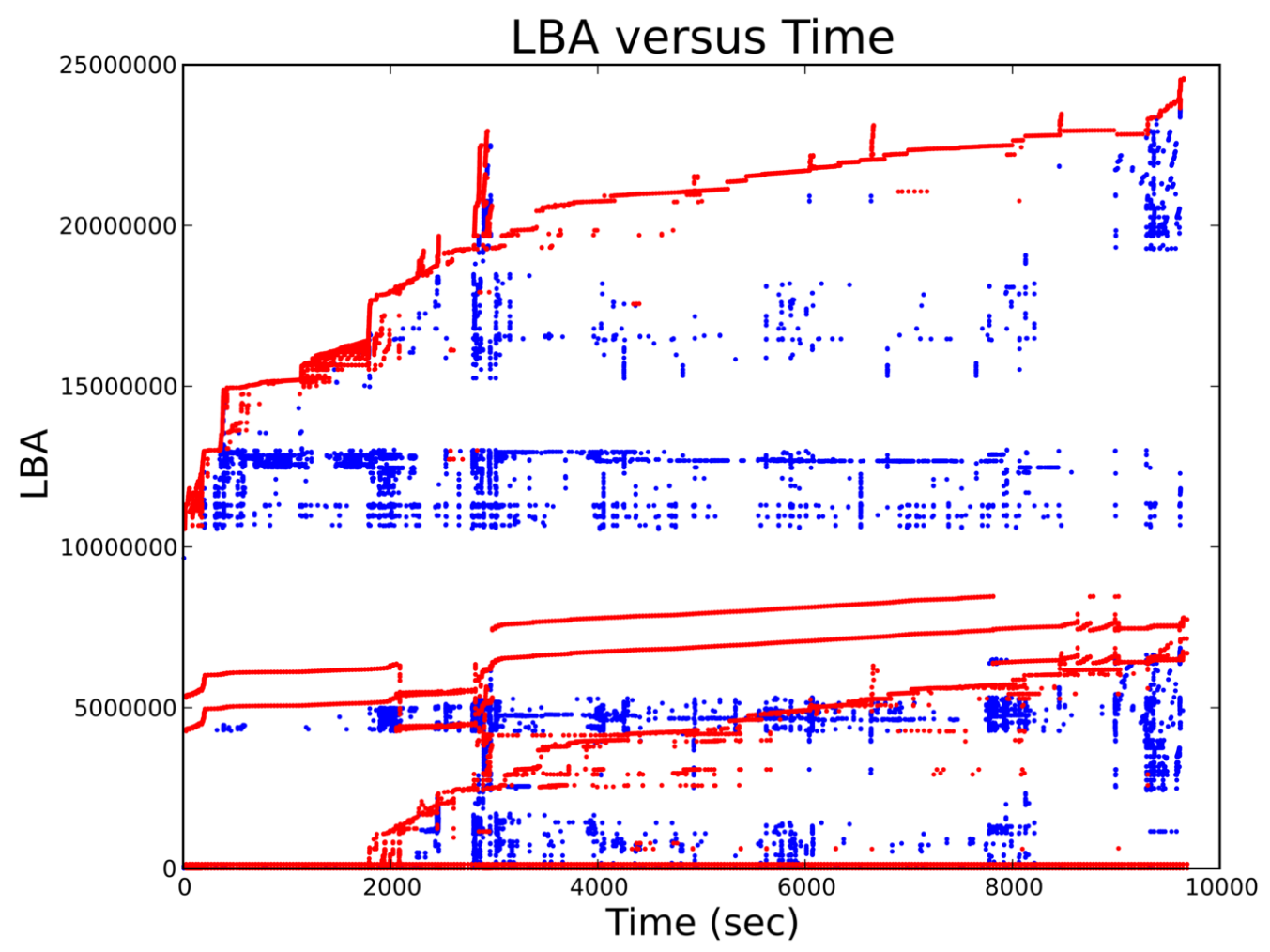

Figure 6.8: Fedora 16 GCC RPM rebuild on Btrfs - LBA 


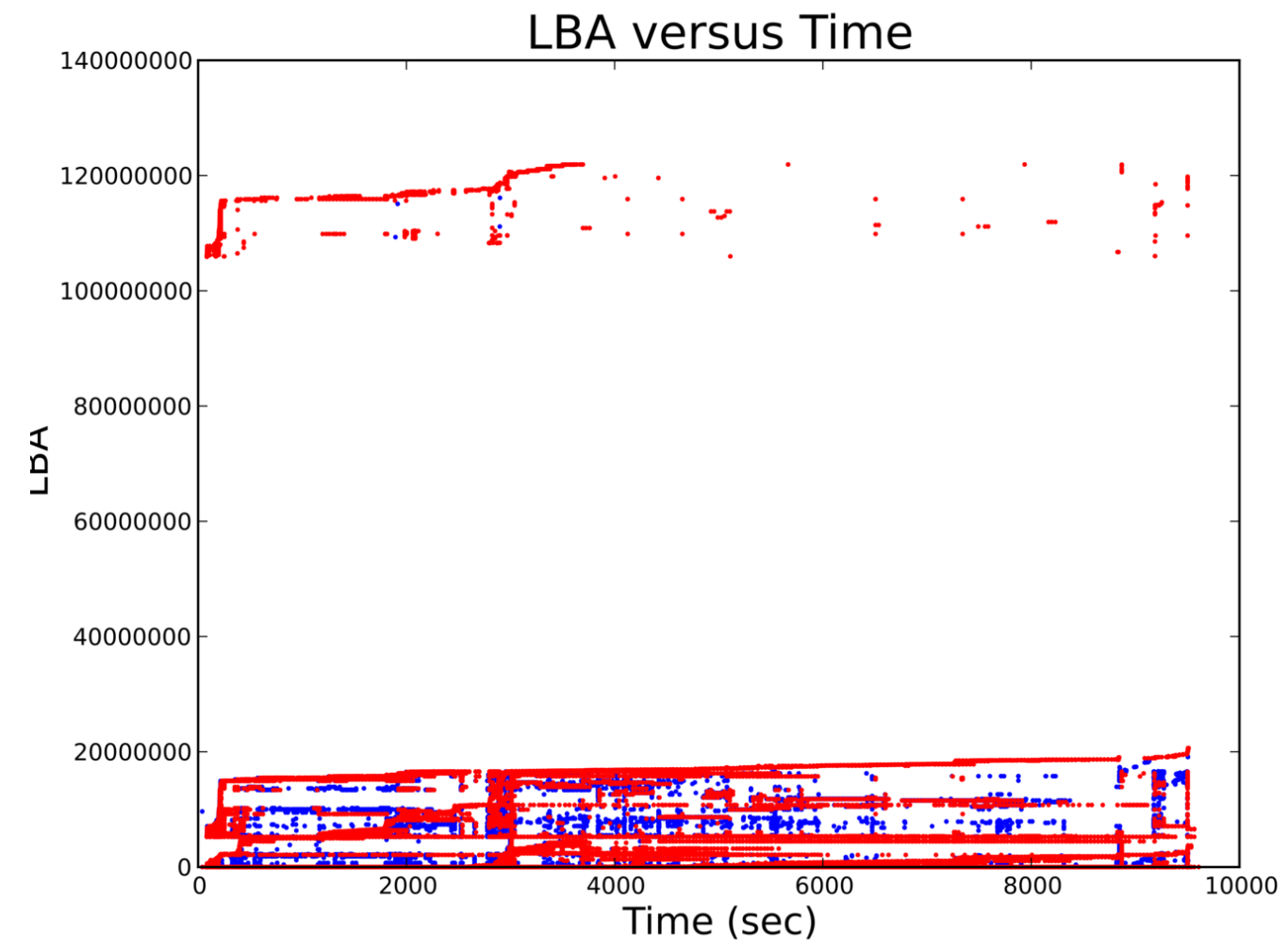

Figure 6.9: Rebuild of Fedora 16 GCC RPM on ext2. LBA as a function of time 


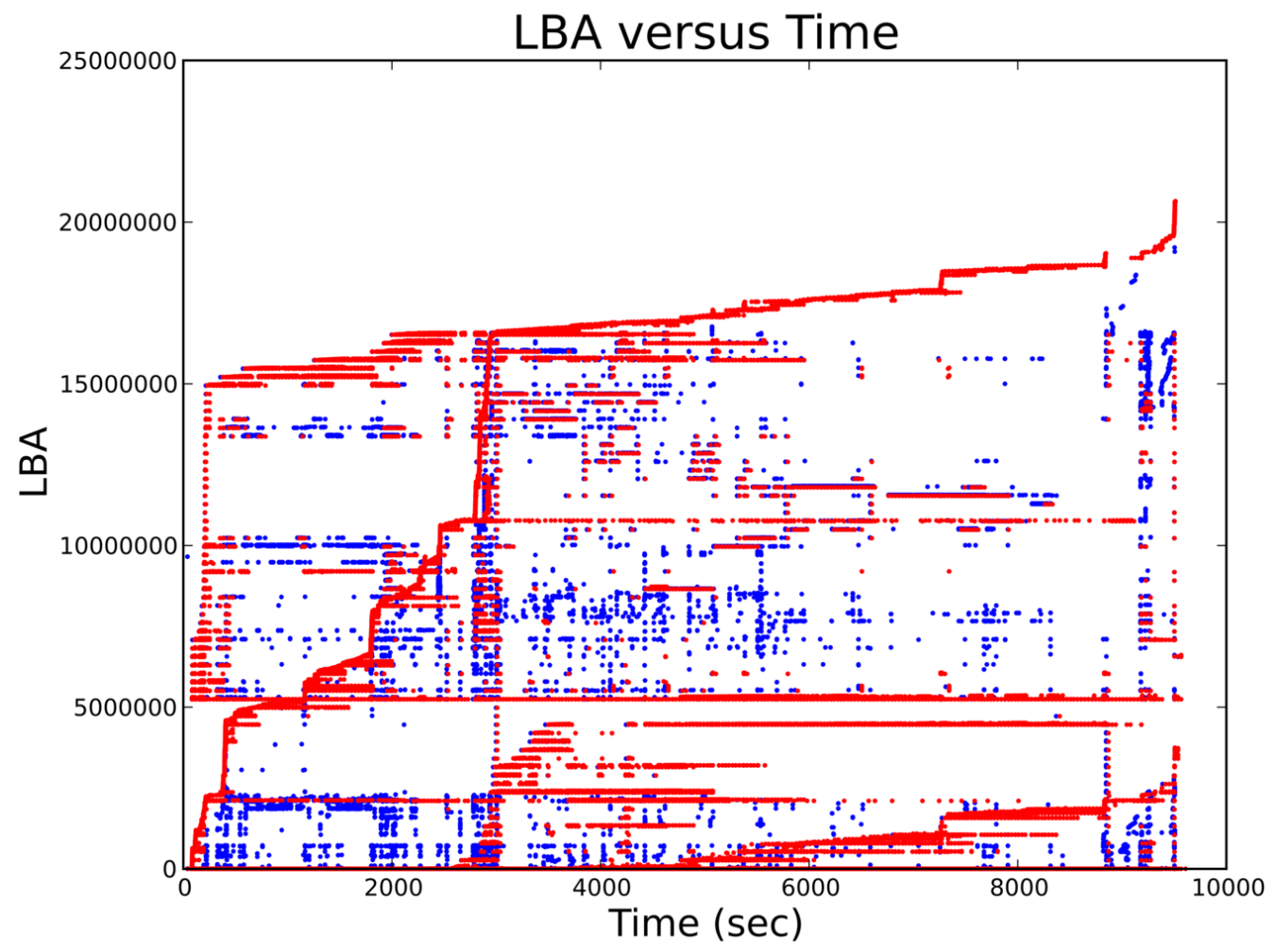

Figure 6.10: Rebuild of Fedora 16 GCC RPM on ext2. LBA range restricted to [0, 25000000] 
commands relative to one another rather than fixed locality on disk. A graph of LBA as a function of time shows what region of the disk is being accessed at any point in time. Given each file system is free to allocate data and metadata in whatever fashion it sees fit, we can and do see differences in the LBA access patterns even with the same workload. The Stream Graph on the other hand focuses on establishing sequential relationships between commands independent of their location on the disk. As long as each file system arranges files as a series of contiguous LBAs then the same workload with the same file access patterns will show the same stream allocation features in the Stream Graph. Figures 6.11 and 6.12 show two different workloads on the same file system. As we would expect the two Stream Graphs are noticeably different. The most succinct description of the result of the Sequential Stream Detection Algorithm on the trace is that the algorithm can be thought of as taking the derivative of the trace. That is the algorithm is a measure of how the access pattern changes with a given workload. 


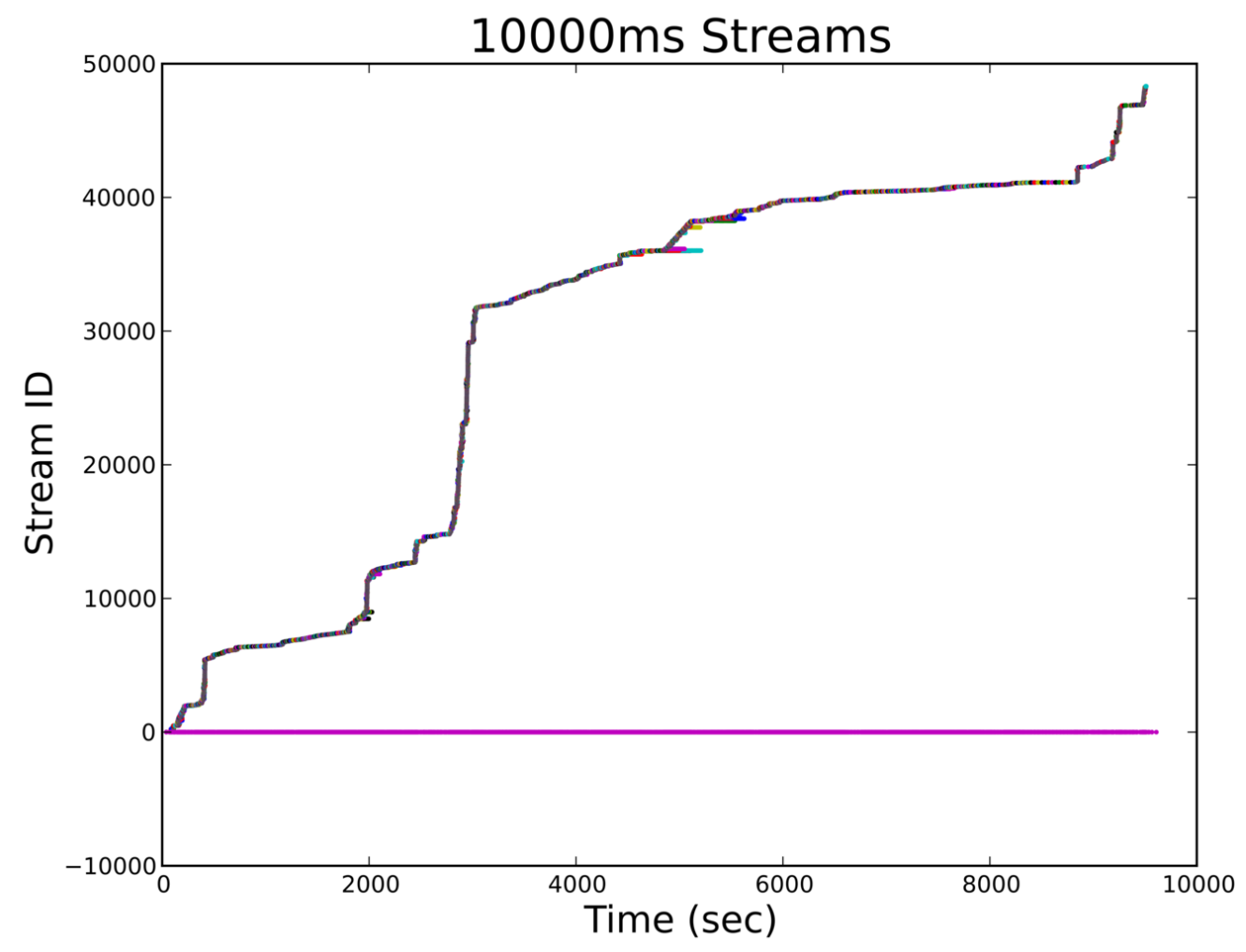

Figure 6.11: Rebuild of Fedora 16 GCC RPM on ext2. Window size 10s 


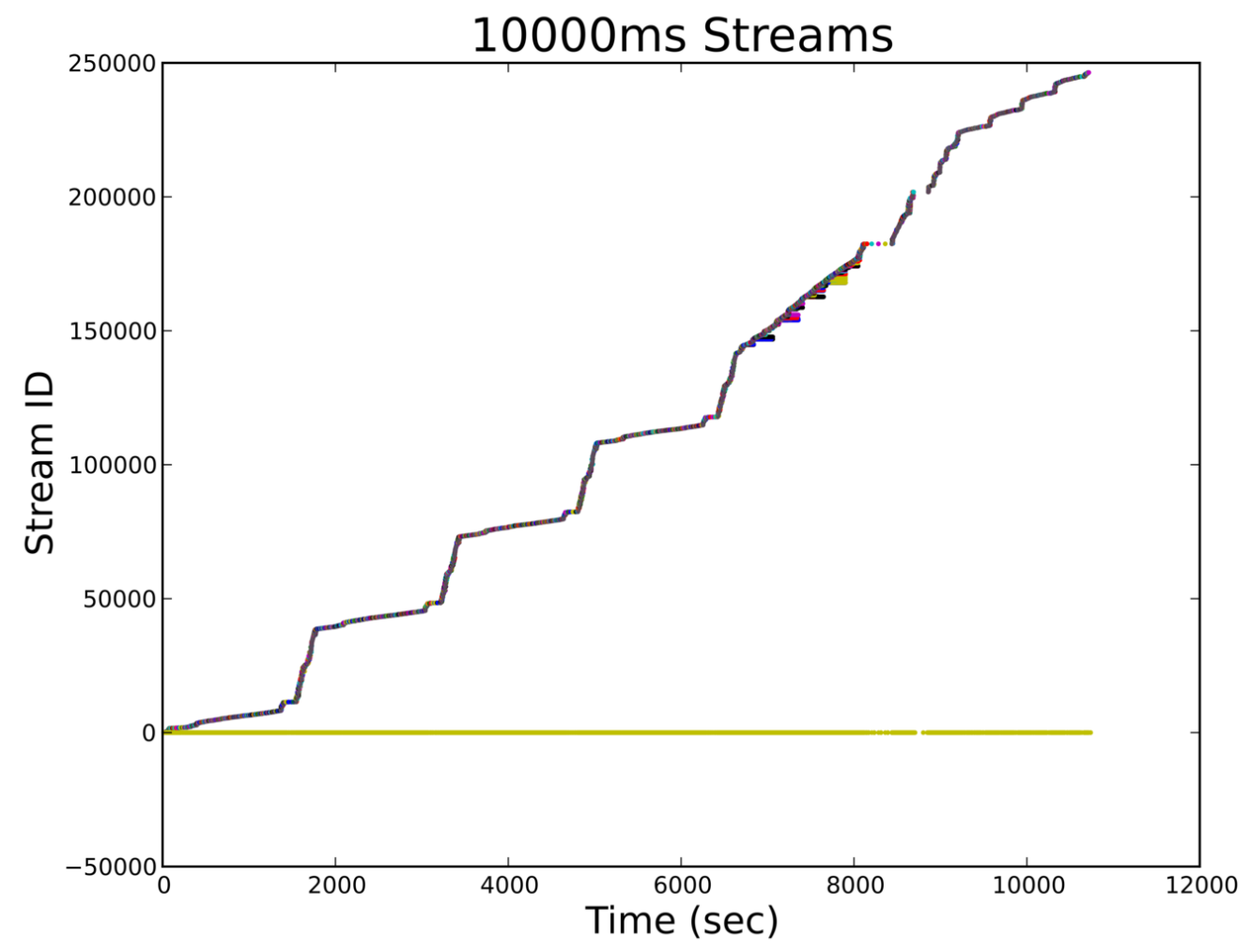

Figure 6.12: Rebuild of Fedora 16 kernel RPM on ext2. Window size 10s 


\section{Chapter 7}

\section{Future Work}

As previously stated, the work presented in this thesis represents a beginning. Hard drive analysis using a serial bus analyzer does not currently occur within academia and the closest research to be found using the technique has been the work of Seagate[15]. Similarly, the Sequential Stream Detection algorithm presented shows promise as a means of identifying and categorizing traces independent of the file system (storage layout). Both areas warrant further exploration.

First and foremost plenty of work remains to further expand PyTrace. PyTrace only supports a subset of SATA commands (commands encountered in the traces taken within the project) and filling in all the commands within the SATA specification represents a large undertaking. PyTrace also only supports SATA which limits the environments with which PyTrace can be used for analysis. For example, business applications running on SAS drives currently cannot be analyzed with PyTrace. The architecture can support SAS but as with supporting the full SATA command set adding SAS represents a respectable amount of work. Finally, PyTrace does not support traces with failed commands which prevents 
analysis of systems with damaged or failing drives which generate IO errors.

Next, there would be value in expanding upon the Sequential Stream Detection algorithm. The algorithm has been vetted against a relatively small set of traces consisting of synthetic IO, media playback, and code compilation. This puts capturing a larger variety of traces, running the Sequential Stream Detection on those traces, and generating Stream Graphs for the traces for analysis at the highest priority for further work. From the standpoint of expanding the algorithm itself, there is currently no tolerance for gaps within a Sequential Stream which was apparent in the mplayer trace. Adding a tolerance for gaps between commands may provide some interesting information and allow for the detection of more streams. Finally, the algorithm as previously mentioned does not split reads and writes into separate streams. While this makes sense in that an application may consider a series of sequential reads and writes as a single stream the drive cannot physically perform both reads and writes at the same time. As such to the drive the reads and writes must be different streams. It would be valuable to modify the implementation to allow for segregating streams into read streams and write streams.

Trace Fingerprinting using the Sequential Stream Detection Algorithm likely represents the most exciting piece of future work. It may be possible to take two fingerprints and from overlaying and stretching the two predict what the fingerprint of running both workloads at the same time would be. It may also be possible to take a system running many applications and demonstrate that one application in particular dominates the drive usage by matching the fingerprint of the running system to that of a single application. This thesis shows the feasibility of such fingerprints for identifying workloads but leaves actual application to future research. 


\section{Chapter 8}

\section{Conclusion}

This thesis has presented a platform for trace analysis using hardware command capture as well as a novel stream detection algorithm built upon the platform. There are existing tools (simulation, modeling, benchmarking, software tracing) for performing such analysis but each of the existing tools have shortcomings which hardware tracing fills. Even with hardware tracing being able to fill the gaps left by existing tools there is little support for analyzing the data produced by hardware tracing. To that end PyTrace was created as a powerful and flexible analysis platform for hardware traces.

The Sequential Stream Detection Algorithm, built upon PyTrace, has shown some interesting information with regard to $d d$ and mplayer. $d d$ is surprisingly bursty in its disk usage while mplayer video playback is less sequential than expected. The less than sequential nature of mplayer would have likely been overlooked if not for the detection algorithm. The algorithm also shows promise as being a tool for identifying workloads in a manner independent of the file system being used. The same workload run on multiple file systems yields Stream Graphs with very similar features while different workloads run on the same file 
system yield Stream Graphs which are noticeably different. No other system is currently known which is capable of uniquely identifying workloads across file systems. 


\section{Bibliography}

[1] A. Allen. Introduction to computer performance analysis with Mathematica. Computer science and scientific computing. AP Professional, 1994.

[2] blktrace(8) - linux man page. http://linux.die.net/man/8/blktrace.

[3] J. S. Bucy and G. R. Ganger. The disksim simulation environment version 3.0 reference manual. Technical report, 2003.

[4] W. Digital. WD VelociRaptor. http://www.wdc.com/wdproducts/ library/SpecSheet/ENG/2879-701284.pdf, 2010.

[5] Y. L. Farrukh, Y. Lu, F. Noman, and D. H. C. Du. Simulation study of iscsi-based storage system*. In In 12th NASA Goddard 83 21st IEEE Conference on Mass Storage Systems and Technologies (MSST2004, pages 399-408, 2004.

[6] IOMeter. http://www.iometer.org/.

[7] JESD. DDR3 SDRAM STANDARD. http://www.jedec.org/sites/ default/files/docs/JESD79-3D.pdf, 2009.

[8] JSON. http://json.org/. 
[9] D. Kotz, S. B. Toh, and S. Radhakrishnan. A detailed simulation model of the hp 97560 disk drive. Technical report, Dept. of Computer Science, Dartmouth College, 1994.

[10] A. Lewis. LVM howto. http://www.tldp.org/HOWTO/LVM-HOWTO/, 2006.

[11] AHCI - OSDev Wiki. http://wiki.osdev.org/ACHI.

[12] J. Ousterhout, H. D. Costa, D. Harrison, J. A. Kunze, M. Kupfer, and J. G. Thompson. A trace-driven analysis of the unix 4.2 bsd file system. pages $15-24,1985$.

[13] PassMark. http://www.harddrivebenchmark.net/.

[14] PCMark05. http://www. futuremark. com/products/pcmark05/.

[15] A. Riska. Disk drive level workload characterization. In In USENIX Annual Conference, 2006.

[16] D. Roselli and T. E. Anderson. A comparison of file system workloads. In In Proceedings of the 2000 USENIX Annual Technical Conference, pages 41-54. USENIX Association, 2000.

[17] C. Ruemmler and J. Wilkes. Unix disk access patterns, 1993.

[18] C. Ruemmler and J. Wilkes. An introduction to disk drive modeling. IEEE Computer, 27:17-28, 1994.

[19] K. A. Smith. Workload-specific file system benchmarks. Technical report, 2001.

[20] C. E. Stevens. Information technology - AT Attachment 8 - ATA/ATAPI Command Set (ATA8-ACS), 2008. 
[21] E. Varki, A. Merchant, J. Xu, and X. Qiu. Issues and challenges in the performance analysis of real disk arrays. IEEE Transactions on Parallel and Distributed Systems, 15:559-574, 2004.

[22] M. Wang, K. Au, A. Ailamaki, A. Brockwell, C. Faloutsos, and G. R. Ganger. Storage device performance prediction with cart models. pages 588-595, 2004.

[23] M. Wang, G. R. Ganger, J. Wilkes, and H. P. Laboratories. Performance modeling of storage devices using machine learning, 2006.

[24] Windows performance analysis. http://msdn.microsoft.com/en-us/ performance/cc825801. 